\title{
Turn-On Mode Fluorescence Switch by Using Negative Photochromic Imidazole Dimer
}

Katsuya Mutoh, Nanae Miyashita, Kaho Arai and Jiro Abe*

Department of Chemistry, School of Science and Engineering, Aoyama Gakuin University, 5-10-1 Fuchinobe, Chuoku, Sagamihara, Kanagawa 252-5258, Japan

E-mail: jiroabe@chem.aoyama.ac.jp

\section{CONTENTS}

1. Syntheses $\quad$ S2

2. ${ }^{1}$ H NMR Spectra $\quad$ S9

3. ${ }^{13}$ C NMR Spectra $\quad$ S15

$\begin{array}{lr}\text { 4. HR-ESI-TOF MS Spectra } & \text { S18 }\end{array}$

5. HPLC Chromatograms $\quad$ S24

6. Fatigue Resistance $\quad$ S25

7. Estimation of Conversion Efficiency $\quad$ S26

$\begin{array}{lr}\text { 8. Fluorescence Lifetime Measurement } & \text { S29 }\end{array}$

9. Cyclic Voltammetry and Rehm-Weller Equation $\quad \mathbf{S 3 2}$

10. Kinetics of Thermal Back Reaction $\quad$ S33

11. Solvent Dependence of Thermal Back Reaction $\quad$ S35

12. References $\quad \mathbf{S 3 6}$ 


\section{Syntheses}

All reactions were monitored by thin-layer chromatography carried out on $0.2 \mathrm{~mm}$ E. Merck silica gel plates $(60 \mathrm{~F}-$ 254). Column chromatography was performed on silica gel (Silica gel $60 \mathrm{~N}$, Kanto Chemical Co., Inc.). NMR spectra were recorded at $400 \mathrm{MHz}$ on a Bruker AVANCE III 400 NanoBay. DMSO- $d_{6}$ and $\mathrm{CDCl}_{3}$ were used as deuterated solvent. ESI-TOF-MS spectra were recorded on a Bruker micrOTOF II-AGA1. Unless otherwise noted, all reagents and reaction solvents were purchased from Tokyo Chemical Industry Co., Ltd., Wako Pure Chemical Industries, Ltd., Sigma-Aldrich Inc. and Kanto Chemical Co., Inc. and were used without further purification.

Scheme S1. Synthetic Scheme of 1
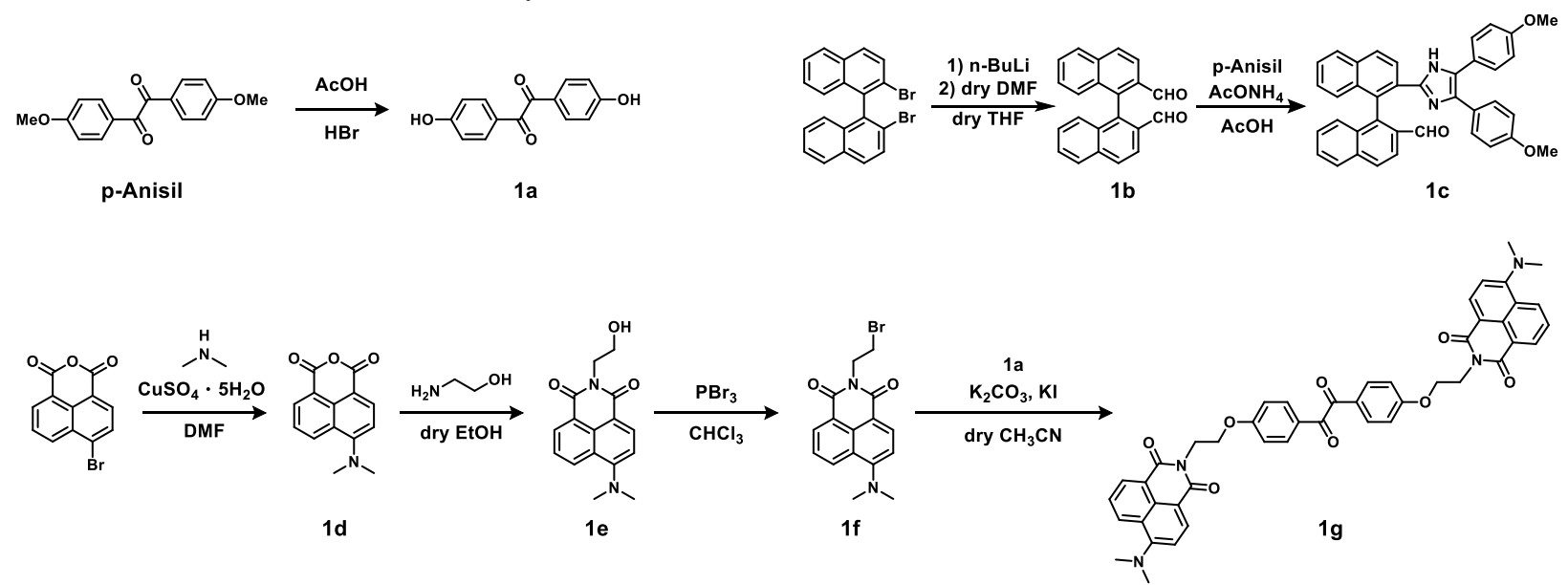

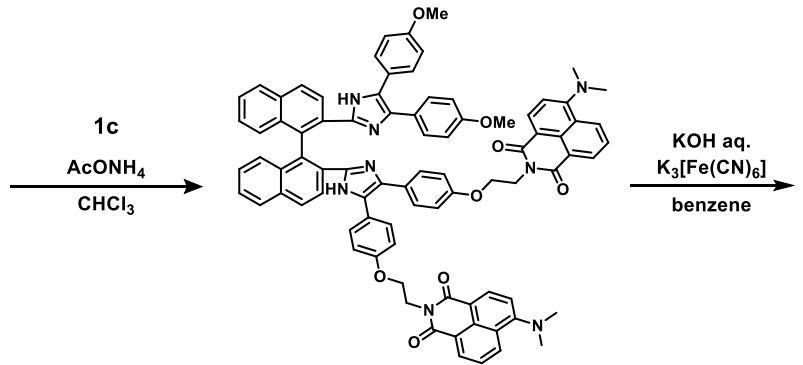

$1 \mathrm{~h}$

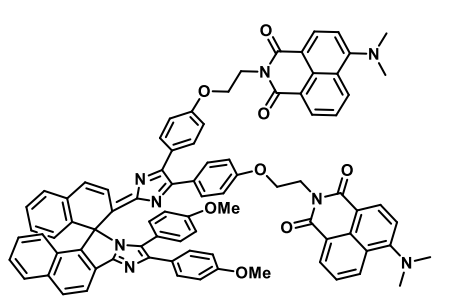

1

4,4'-dihydroxy-benzil (1a)

p-Anisil (1.00 g, $3.70 \mathrm{mmol})$, $\mathrm{HBr}$ aq. (8 $\mathrm{mL}, 47.0-49.0 \%)$ and acetic acid $(8 \mathrm{~mL})$ were added to a round bottom flask and the mixture was refluxed for 21 hours. After quenching with water, the reaction mixture was extracted with AcOEt. The organic extract was washed with water and brine, and passed through a phase separator paper.. After removal of the solvents, the crude product was

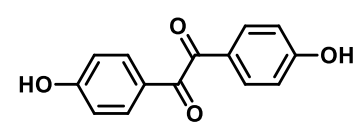

$1 \mathrm{a}$ purified by silica gel column chromatography $(\mathrm{AcOEt} / \mathrm{hexane}=1 / 1)$, to give the desired product as a pale yellow solid (672 mg, $75 \%$ ). ${ }^{1} \mathrm{H}$ NMR (400 MHz, DMSO- $\left.d_{6}\right) \delta: 10.84(\mathrm{br}, 2 \mathrm{H}), 7.75-7.72(\mathrm{~m}, 4 \mathrm{H}), 6.94-6.90$ (m, 4H); HRMS (ESI-TOF) calculated for $\mathrm{C}_{14} \mathrm{H}_{10} \mathrm{O}_{4}[\mathrm{M}+\mathrm{Na}]^{+}: 265.0471$, found: 265.0464 . 


\section{[1,1'-Binaphthalene]-2,2'-dicarboxaldehyde (1b)}

1.6 M n-BuLi in hexane $(12.1 \mathrm{~mL}, 19.4 \mathrm{mmol})$ was added dropwise into a solution of 2,2'dibromo-1,1'-binaphthyl $(2.104 \mathrm{~g}, 5.104 \mathrm{mmol})$ in $20 \mathrm{~mL}$ of dry THF at $-78{ }^{\circ} \mathrm{C}$ under $\mathrm{N}_{2}$ atmosphere. The reaction mixture was stirred at $-78^{\circ} \mathrm{C}$ for 1 hour. Dry DMF $(1.4 \mathrm{~mL}, 15 \mathrm{mmol})$ was added dropwise to the solution, and the mixture was then stirred at $-78^{\circ} \mathrm{C}$ for 1 hour. The reaction mixture was slowly warmed to

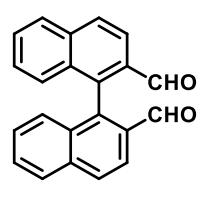

$1 \mathbf{b}$ room temperature, and stirred for 15 hours. After quenching with $1 \mathrm{~N} \mathrm{HCl} \mathrm{aq.,} \mathrm{the} \mathrm{reaction} \mathrm{mixture} \mathrm{was}$ extracted with $\mathrm{CH}_{2} \mathrm{Cl}_{2}$. The organic extract was washed with water and brine, and passed through a phase separator paper. After removal of the solvents, the crude product was purified by silica gel column chromatography $\left(\mathrm{CH}_{2} \mathrm{Cl}_{2} / \mathrm{hexane}=3 / 2\right)$, to give the desired product as a white powder (1.055 g, $67 \%) .{ }^{1} \mathrm{H}$ NMR (400 MHz, DMSO- $\left.d_{6}\right) \delta: 9.57(\mathrm{~s}, 2 \mathrm{H}), 8.33(\mathrm{~d}, J=8.6 \mathrm{~Hz}, 2 \mathrm{H}), 8.20$ $(\mathrm{d}, J=8.1 \mathrm{~Hz}, 2 \mathrm{H}), 8.13(\mathrm{~d}, J=8.6 \mathrm{~Hz}, 2 \mathrm{H}), 7.73(\mathrm{~m}, 2 \mathrm{H}), 7.46(\mathrm{~m}, 2 \mathrm{H}), 7.09$ (d, $J=8.4 \mathrm{~Hz}, 2 \mathrm{H})$.

\section{2'-(4,5-bis(4-methoxyphenyl)-1 $\mathrm{H}$-imidazol-2-yl)-[1,1'-binaphthalene]-2-carbaldehyde (1c)}

[1,1'-Binaphthalene]-2,2'-dicarboxaldehyde (1b) (303 mg, $0.976 \mathrm{mmol}), p$-Anisil $(241 \mathrm{mg}, 0.890 \mathrm{mmol})$ and ammonium acetate $(1.66 \mathrm{~g}, 21.5 \mathrm{mmol})$ were dissolved in acetic acid $(18 \mathrm{~mL})$. The mixture was stirred at $110{ }^{\circ} \mathrm{C}$ for 19 hours. After cooling to room temperature, the reaction mixture was neutralized with aqueous $\mathrm{NH}_{3}$, and the reaction mixture was extracted with AcOEt. The combined organic layer was washed with water and brine, and passed through a

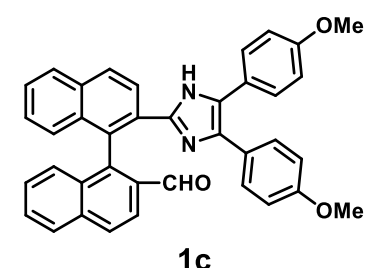

$1 \mathrm{c}$ phase separator paper. After removal of the solvents, the crude product was purified by silica gel column chromatography (AcOEt $/$ Hexane $=1 / 2)$ to give the desired product as a pale brown crystal $(125 \mathrm{mg}, 25 \%) .{ }^{1} \mathrm{H}$ NMR $(400$ MHz, DMSO-d $\left.d_{6}\right) \delta: 12.51(\mathrm{~s}, 1 \mathrm{H}), 9.72(\mathrm{~s}, 1 \mathrm{H}), 8.28(\mathrm{~s}, 1 \mathrm{H}), 8.20(\mathrm{~d}, J=8.7 \mathrm{~Hz}, 1 \mathrm{H}), 8.12-8.09(\mathrm{~m}, 3 \mathrm{H}), 7.61-7.53(\mathrm{~m}, 2 \mathrm{H}), 7.37-$ $7.27(\mathrm{~m}, 4 \mathrm{H}), 7.09$ (d, $J=8.5 \mathrm{~Hz}, 1 \mathrm{H}), 7.0-6.94(\mathrm{~m}, 3 \mathrm{H}), 6.85$ (d, $J=8.8 \mathrm{~Hz}, 2 \mathrm{H}), 6.65$ (d, J=8.8 Hz, 2H), 3.77 (s, $3 \mathrm{H}), 3.68$ (s, 3H). ${ }^{13} \mathrm{C}$ NMR (100 MHz, $\left.\mathrm{CDCl}_{3}\right) \delta: 191.88,170.31,158.80,157.63,144.24,143.88,135.83,135.81,133.76,132.71,132.60$, $132.25,129.73,129.56,128.94,128.57,128.30,128.21,128.18,128.15,127.39,127.28,127.12,126.95,126.45,126.03,125.16$, 123.18, 121.43, 113.99, 113.19, 59.73, 55.13, 54.90, 20.73, 14.05; HRMS (ESI-TOF) calculated for $\mathrm{C}_{38} \mathrm{H}_{28} \mathrm{~N}_{2} \mathrm{O}_{3}[\mathrm{M}+\mathrm{H}]^{+}: 561.2173$, found: 561.2158 .

\section{4-(dimethylamino)-1,8-naphthalic anhydride (1d)}

4-bromo-naphthalic anhydride $(4.006 \mathrm{~g}, 14.46 \mathrm{mmol})$, dimethylamine $(6.4 \mathrm{~mL}, 48 \mathrm{mmol})$ and $\mathrm{CuSO}_{4} \cdot 5 \mathrm{H}_{2} \mathrm{O}(524 \mathrm{mg}, 3.28 \mathrm{mmol})$ were added to DMF $(24 \mathrm{~mL})$ in a round bottom flask and the reaction mixture was refluxed at $140{ }^{\circ} \mathrm{C}$ for 3 hours. After quenching with water, the precipitate was collected and dried. The solid was dissolved in chloroform and filtered to remove insoluble impurities. After removal of the solvents, the crude product was purified by silica gel column chromatography $\left(\mathrm{CHCl}_{3} / \mathrm{hexane}=1 / 1\right)$, to give the desired

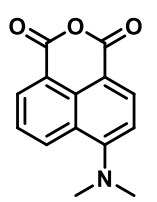

1d product as a yellow solid (2.79 g, $80 \%) .{ }^{1} \mathrm{H}$ NMR $\left(400 \mathrm{MHz}, \mathrm{CDCl}_{3}\right) \delta: 8.54(\mathrm{dd}, J=7.3 \mathrm{~Hz}, J=1.1 \mathrm{~Hz}, 1 \mathrm{H})$, $8.48(\mathrm{dd}, J=8.5 \mathrm{~Hz}, J=1.0 \mathrm{~Hz}, 1 \mathrm{H}), 8.43(\mathrm{~d}, J=8.3 \mathrm{~Hz}, 1 \mathrm{H}), 7.67(\mathrm{~d}, J=8.4 \mathrm{~Hz}, J=7.3 \mathrm{~Hz}, 1 \mathrm{H}), 7.10(\mathrm{~d}, J=8.4 \mathrm{~Hz}, 1 \mathrm{H}), 3.19$ $(\mathrm{s}, 6 \mathrm{H})$; HRMS (ESI-TOF) calculated for $\mathrm{C}_{14} \mathrm{H}_{11} \mathrm{NO}_{3}[\mathrm{M}+\mathrm{H}]^{+}: 242.0812$, found: 242.0805 . 


\section{6-(dimethylamino)-2-(2-hydroxyethyl)-1H-benzo[de]isoquinoline-1,3(2H)-dione (1e)}

4-(dimethylamino)-1,8-naphthalic anhydride (1d) (1.004 g, $4.163 \mathrm{mmol})$ was placed in a round bottom flask under $\mathrm{N}_{2}$ atmosphere. Dry ethanol $(27 \mathrm{~mL})$ was added to the flask and a clear orange solution formed. After heating to $80^{\circ} \mathrm{C}$, ethanolamine $(0.32 \mathrm{~mL}, 5.3 \mathrm{mmol})$ was added. The mixture was refluxed for another 2 hours and the solution was allowed to cool to room temperature. After removal of the solvents, the desired product was obtained as an orange solid (1.284 g, quantitative). ${ }^{1} \mathrm{H}$ NMR (400 MHz, $\left.\mathrm{CDCl}_{3}\right) \delta: 8.59$ (dd, $J=7.3 \mathrm{~Hz}, J=1.1 \mathrm{~Hz}, 1 \mathrm{H}), 8.49(\mathrm{~d}, J=8.2 \mathrm{~Hz}, 1 \mathrm{H}), 8.46(\mathrm{dd}, J=8.5 \mathrm{~Hz}, J=1.2 \mathrm{~Hz}, 1 \mathrm{H}), 7.67(\mathrm{dd}, J=7.9 \mathrm{~Hz}$, $J=7.3 \mathrm{~Hz}, 1 \mathrm{H}), 7.13(\mathrm{~d}, J=8.2 \mathrm{~Hz}, 1 \mathrm{H}), 4.60$ (t, $J=7.2 \mathrm{~Hz}, 2 \mathrm{H}), 3.67$ (t, $J=7.2 \mathrm{~Hz}, 2 \mathrm{H}), 3.12(\mathrm{~s}, 6 \mathrm{H})$; HRMS

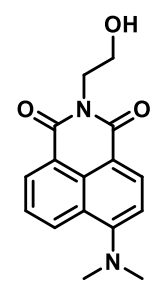

1e

(ESI-TOF) calculated for $\mathrm{C}_{16} \mathrm{H}_{16} \mathrm{~N}_{2} \mathrm{O}_{3}[\mathrm{M}+\mathrm{Na}]^{+}: 307.1053$, found: 307.1042 .

\section{2-(2-bromoethyl)-6-(dimethylamino)-1H-benzo[de]isoquinoline-1,3(2H)-dione (1f)}

To a solution of 6-(dimethylamino)-2-(2-hydroxyethyl)-1H-benzo[de $]$ isoquinoline-1,3(2H)-dione (1e) $(1.150 \mathrm{mg}, 4.059 \mathrm{mmol})$ in $\mathrm{CHCl}_{3} 23 \mathrm{~mL}$ at $0{ }^{\circ} \mathrm{C}$ was added dropwise phosphorus tribromide $(1.4 \mathrm{~mL}, 15$ $\mathrm{mmol})$. The reaction mixture was slowly warmed to room temperature, and stirred for 3 minutes. After removal of the solvent in vacuo, ice and $\mathrm{K}_{2} \mathrm{CO}_{3}$ aq. $(2 \mathrm{M}, 10 \mathrm{~mL})$ were added to the crude product. The mixture was extracted with $\mathrm{CH}_{2} \mathrm{Cl}_{2}$. The organic extract was washed with water and brine, and passed through a phase separator paper. After removal of the solvents, the crude product was purified by silica gel column

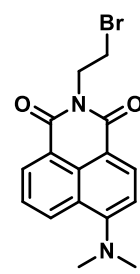

$1 f$ chromatography $(\mathrm{AcOEt} / \mathrm{hexane}=1 / 2)$, to give desired product as a yellow solid $(760 \mathrm{mg}, 54 \%) .{ }^{1} \mathrm{H}$ NMR $(400$ $\left.\mathrm{MHz}, \mathrm{CDCl}_{3}\right) \delta: 8.59(\mathrm{dd}, J=7.3 \mathrm{~Hz}, J=1.1 \mathrm{~Hz}, 1 \mathrm{H}), 8.49(\mathrm{~d}, J=8.2 \mathrm{~Hz}, 1 \mathrm{H}), 8.46(\mathrm{dd}, J=8.5 \mathrm{~Hz}, J=1.1 \mathrm{~Hz}, 1 \mathrm{H}), 7.67(\mathrm{dd}, J$ $=8.4 \mathrm{~Hz}, J=7.4 \mathrm{~Hz}, 1 \mathrm{H}), 7.13(\mathrm{~d}, J=8.2 \mathrm{~Hz}, 1 \mathrm{H}), 4.60(\mathrm{t}, J=7.2 \mathrm{~Hz}, 2 \mathrm{H}), 3.66(\mathrm{t}, J=7.2 \mathrm{~Hz}, 2 \mathrm{H}), 3.12$ (s, 6H); HRMS (ESITOF) calculated for $\mathrm{C}_{16} \mathrm{H}_{15} \mathrm{BrN}_{2} \mathrm{O}_{2}[\mathrm{M}+\mathrm{Na}]^{+}: 369.0209$, found: 369.0194 .

\section{2,2'-(((oxalylbis(4,1-phenylene))bis(oxy))bis(ethane-2,1-diyl))bis(6-(dimethylamino)-1H-benzo[de]isoquinoline-1,3(2H)-}

dione) (1g)

2-(2-bromoethyl)-6-(dimethylamino)-1H-benzo[de]isoquinoline1,3(2H)-dione (1f) (430 mg, $1.24 \mathrm{mmol}), 4,4$ '-dihydroxy-benzil (1a) (112 mg, $0.464 \mathrm{mmol}), \mathrm{K}_{2} \mathrm{CO}_{3}(359 \mathrm{mg}, 2.59 \mathrm{mmol})$ and $\mathrm{KI}(43 \mathrm{mg}, 0.26 \mathrm{mmol})$ were add to a round bottom flask under $\mathrm{N}_{2}$ atmosphere. The mixture was dissolved in dry $\mathrm{CH}_{3} \mathrm{CN}(30 \mathrm{~mL})$ and stirred at $80^{\circ} \mathrm{C}$ for 25 hours. After cooling to room temperature, the yellow precipitate was collected and dried. The precipitate was dissolved in $\mathrm{CHCl}_{3}$ and filtered to remove insoluble impurities. The

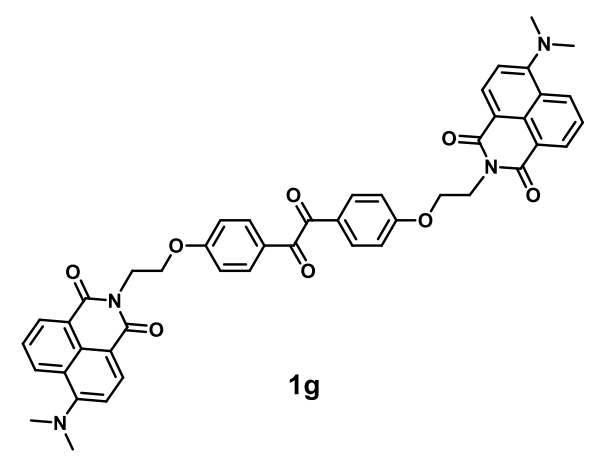
organic layer was washed with water and brine, and passed through a phase separator paper. The solvent was evaporated to give desired product as a pale yellow powder (321 mg, $89 \%$ ). ${ }^{1} \mathrm{H} \mathrm{NMR}(400 \mathrm{MHz}$, $\left.\mathrm{CDCl}_{3}\right) \delta: 8.58(\mathrm{dd}, J=7.3 \mathrm{~Hz}, J=1.0 \mathrm{~Hz}, 2 \mathrm{H}), 8.49(\mathrm{~d}, J=8.2 \mathrm{~Hz}, 2 \mathrm{H}), 8.45(\mathrm{dd}, J=8.5 \mathrm{~Hz}, J=1.0 \mathrm{~Hz}, 2 \mathrm{H}), 7.87$ (m, $\left.4 \mathrm{H}\right), 7.66$ $(\mathrm{dd}, J=8.4 \mathrm{~Hz}, J=7.4 \mathrm{~Hz}, 2 \mathrm{H}), 7.12(\mathrm{~d}, J=8.2 \mathrm{~Hz}, 2 \mathrm{H}), 7.00(\mathrm{~m}, 4 \mathrm{H}), 4.63(\mathrm{t}, J=6.3 \mathrm{~Hz}, 4 \mathrm{H}), 4.38(\mathrm{t}, J=6.4 \mathrm{~Hz}, 4 \mathrm{H}), 3.12(\mathrm{~s}$, 12H). ${ }^{13} \mathrm{C}$ NMR (100 MHz, $\left.\mathrm{CDCl}_{3}\right) \delta: 193.53,164.68,164.06,163.90,157.24,132.95,132.31,131.55,131.30,130.39,126.34$, 125.23, 124.88, 122.73, 114.87, 114.43, 113.28, 64.98, 44.76, 38.54; HRMS (ESI-TOF) calculated for $\mathrm{C}_{46} \mathrm{H}_{38} \mathrm{~N}_{4} \mathrm{O} 8$ [M+Na] $]^{+}$ 
797.2582, found: 797.2571 .

\section{2,2'-(((2-(2'-(4,5-bis(4-methoxyphenyl)-1H-imidazol-2-yl)-[1,1'-binaphthalen]-2-yl)-1H-imidazole-4,5-diyl)bis(4,1-}

phenylene))bis(oxy))bis(ethane-2,1-diyl))bis(6-(dimethylamino)-1H-benzo[de]isoquinoline-1,3(2H)-dione) (1h)

2'-(4,5-bis(4-methoxyphenyl)-1H-imidazol-2-yl)-[1,1'-

binaphthalene]-2-carbaldehyde(1c) (43 mg, $0.076 \mathrm{mmol}), 2,2^{\prime}-((($ oxalylbis $(4,1-$ phenylene))bis(oxy))bis(ethane-2,1-diyl))bis(6-(dimethylamino)-1H-

benzo[de]isoquinoline-1,3(2H)-dione) $(1 \mathrm{~g})(72 \mathrm{mg}, 0.093 \mathrm{mmol})$ and ammonium acetate $(284 \mathrm{mg}, 3.69 \mathrm{mmol})$ were dissolved in $\mathrm{CHCl}_{3}(5 \mathrm{~mL})$. The mixture was stirred at $110{ }^{\circ} \mathrm{C}$ for 11 hours. After cooling to room temperature, the reaction mixture was added water and extracted with $\mathrm{CHCl}_{3}$. The organic extract was washed with water and brine, and passed through a phase separator paper. The product was purified by silica gel column chromatography $\left(\mathrm{AcOEt} / \mathrm{CH}_{2} \mathrm{Cl}_{2}=2 / 3\right.$ ) to give the desired product as a yellow solid (55 mg, $55 \%) .{ }^{1} \mathrm{H}$ NMR (400 MHz,

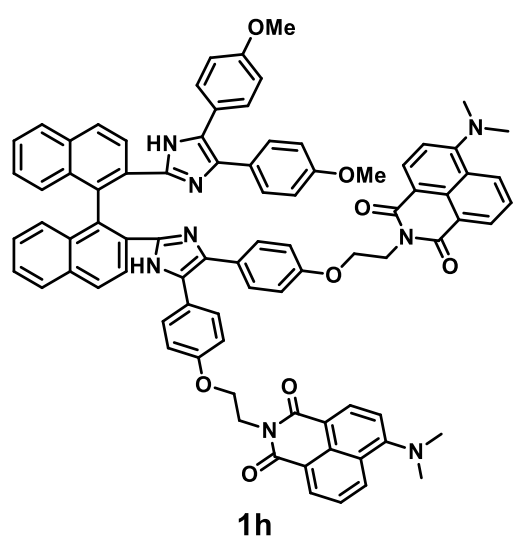
DMSO-d6) $\delta: 13.81(\mathrm{~s}, 2 \mathrm{H}), 8.45$ (dd, $J=14.0 \mathrm{~Hz}, J=7.9 \mathrm{~Hz}, 4 \mathrm{H}), 8.32(\mathrm{~d}, J=8.2 \mathrm{~Hz}, 2 \mathrm{H}), 8.16-8.14(\mathrm{~m}, 2 \mathrm{H}), 7.99(\mathrm{dt}, J=8.2$ $\mathrm{Hz}, J=3.2 \mathrm{~Hz}, 4 \mathrm{H}), 7.74-7.70(\mathrm{~m}, 2 \mathrm{H}), 7.47-7.43(\mathrm{~m}, 2 \mathrm{H}), 7.12(\mathrm{~d}, J=8.4 \mathrm{~Hz}, 2 \mathrm{H}), 7.13-7.04(\mathrm{~m}, 8 \mathrm{H}), 6.89(\mathrm{~d}, J=8.6 \mathrm{~Hz}, 2 \mathrm{H})$, $6.82(\mathrm{t}, J=8.4 \mathrm{~Hz}, 4 \mathrm{H}), 6.71(\mathrm{dd}, J=8.7 \mathrm{~Hz}, J=6.8 \mathrm{~Hz}, 4 \mathrm{H}), 4.40-4.35(\mathrm{~m}, 4 \mathrm{H}), 4.19-4.12(\mathrm{~m}, 4 \mathrm{H}), 3.69(\mathrm{~s}, 3 \mathrm{H}), 3.65(\mathrm{~s}, 3 \mathrm{H})$, 3.06 (s, 12H). ${ }^{13} \mathrm{C}$ NMR (100 MHz, $\left.\mathrm{CDCl}_{3}\right) \delta: 163.67,162.96,158.50,157.85,157.46,156.81,156.57,146.44,146.38,153.21$, $134.35,134.28,132.95,132.44,132.34,131.61,130.62,130.13,129.62,128.65,128.15,127.88,127.29,127.09,126.89,126.44$, $125.63,124.88,124.06,123.24,123.04,122.09,114.57,114.06,113.53,112.96,112.85,64.19,64.08,55.01,54.89,44.28,38.15$; HRMS (ESI-TOF) calculated for $\mathrm{C}_{84} \mathrm{H}_{66} \mathrm{~N}_{8} \mathrm{O}_{8}[\mathrm{M}+\mathrm{H}]^{+}: 1315.5076$, found: 1315.5109 .

\section{Compound 1}

All manipulations were carried out with the exclusion of light. Under nitrogen, to a solution of 2,2'-((((2-(2'-(4,5-bis(4-methoxyphenyl)$1 H$-imidazol-2-yl)-[1,1'-binaphthalen]-2-yl)-1H-imidazole-4,5-diyl)bis(4,1phenylene))bis(oxy))bis(ethane-2,1-diyl))bis(6-(dimethylamino)-1H-

benzo[de]isoquinoline-1,3(2H)-dione) (1h) (35 mg, $0.027 \mathrm{mmol})$ in benzene $(26 \mathrm{~mL})$ was added a solution of potassium ferricyanide (794 mg, $2.41 \mathrm{mmol}$ ) and $\mathrm{KOH}(270 \mathrm{mg}, 4.81 \mathrm{mmol})$ in water $(21 \mathrm{~mL})$, and the reaction mixture was vigorously stirred for 4 hours. The organic layer was separated, washed with water, and concentrated. The crude product was purified with silica gel

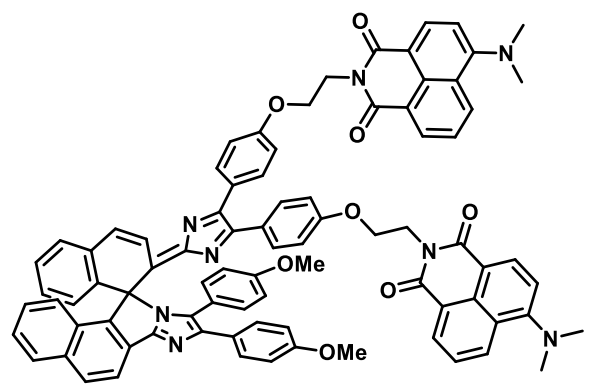

1

column chromatography $\left(\mathrm{CH}_{2} \mathrm{Cl}_{2} / \mathrm{AcOEt}=5 / 1\right.$ to $\left.2 / 1\right)$ and reverse phase $\mathrm{HPLC}\left(\mathrm{CH}_{3} \mathrm{CN} / \mathrm{THF}=50 / 1\right)$ to yield the desired product as a dark red solid (15 mg, $41 \%) .{ }^{1} \mathrm{H}$ NMR (400 MHz, DMSO- $\left.d_{6}\right) \delta: 8.50-8.42(\mathrm{~m}, 4 \mathrm{H}), 8.36-8.30(\mathrm{~m}, 2 \mathrm{H}), 8.11(\mathrm{dd}, J=8.4 \mathrm{~Hz}$, $J=1.8 \mathrm{~Hz}, 1 \mathrm{H}), 8.03(\mathrm{t}, J=7.6 \mathrm{~Hz}, 1 \mathrm{H}), 7.94(\mathrm{dd}, J=8.0 \mathrm{~Hz}, J=3.2 \mathrm{~Hz}, 1 \mathrm{H}), 7.80(\mathrm{dd}, J=9.8 \mathrm{~Hz}, J=5.9 \mathrm{~Hz}, 1 \mathrm{H}), 7.76-7.69$ $(\mathrm{m}, 2 \mathrm{H}), 7.51(\mathrm{~d}, J=7.5 \mathrm{~Hz}, 1 \mathrm{H}), 7.45-7.27(\mathrm{~m}, 10 \mathrm{H}), 7.22-7.13(\mathrm{~m}, 4 \mathrm{H}), 6.95-6.89(\mathrm{~m}, 4 \mathrm{H}), 6.83-6.77(\mathrm{~m}, 3 \mathrm{H}), 6.60(\mathrm{dd}, J=$ $8.6 \mathrm{~Hz}, J=7.3 \mathrm{~Hz}, 2 \mathrm{H}), 6.29(\mathrm{t}, J=8.6 \mathrm{~Hz}, 2 \mathrm{H}), 4.43-4.34(\mathrm{~m}, 4 \mathrm{H}), 4.31-4.25(\mathrm{~m}, 2 \mathrm{H}), 4.15-4.10(\mathrm{~m}, 2 \mathrm{H}), 3.77(\mathrm{~d}, J=15.1 \mathrm{~Hz}$, 
$6 \mathrm{H}), 3.66(\mathrm{~d}, J=3.0 \mathrm{~Hz}, 12 \mathrm{H}) .{ }^{13} \mathrm{C} \mathrm{NMR}\left(100 \mathrm{MHz}, \mathrm{CDCl}_{3}\right) \delta: 163.93,163.82,163.74,163.70,163.66,163.52,162.99,161.57$, $161.34,160.84,160.25,159.79,158.90,157.63,156.62,152.40,152.34,151.20,151.12,140.89,137.98,136.51,133.09,132.38$, $131.66,131.31,131.15,130.75,130.66,130.12$, 129.67, 127.73, 127.32, 127.10, 125.72, 125.39, 125.22, 125.19, 124.93, 124.61, $124.08,122.18,122.13,122.10,114.25,114.08,113.83,113.74,113.54,112.97,112.88,65.80,64.37,59.72,55.36,55.24,55.01$, 54.89, 44.32, 44.30, 38.07, 20.73, 14.05; HRMS (ESI-TOF) calculated for $\mathrm{C}_{84} \mathrm{H}_{64} \mathrm{~N}_{8} \mathrm{O} 8$ [M+H] $]^{+}: 1313.4920$, found: 1313.4911.

Scheme S2. Synthetic Scheme of 2
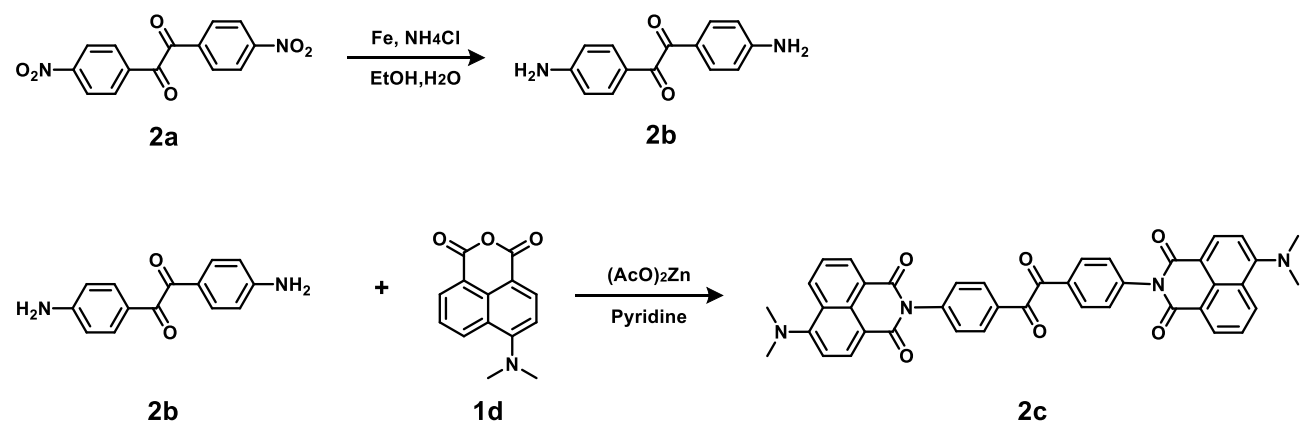

2b
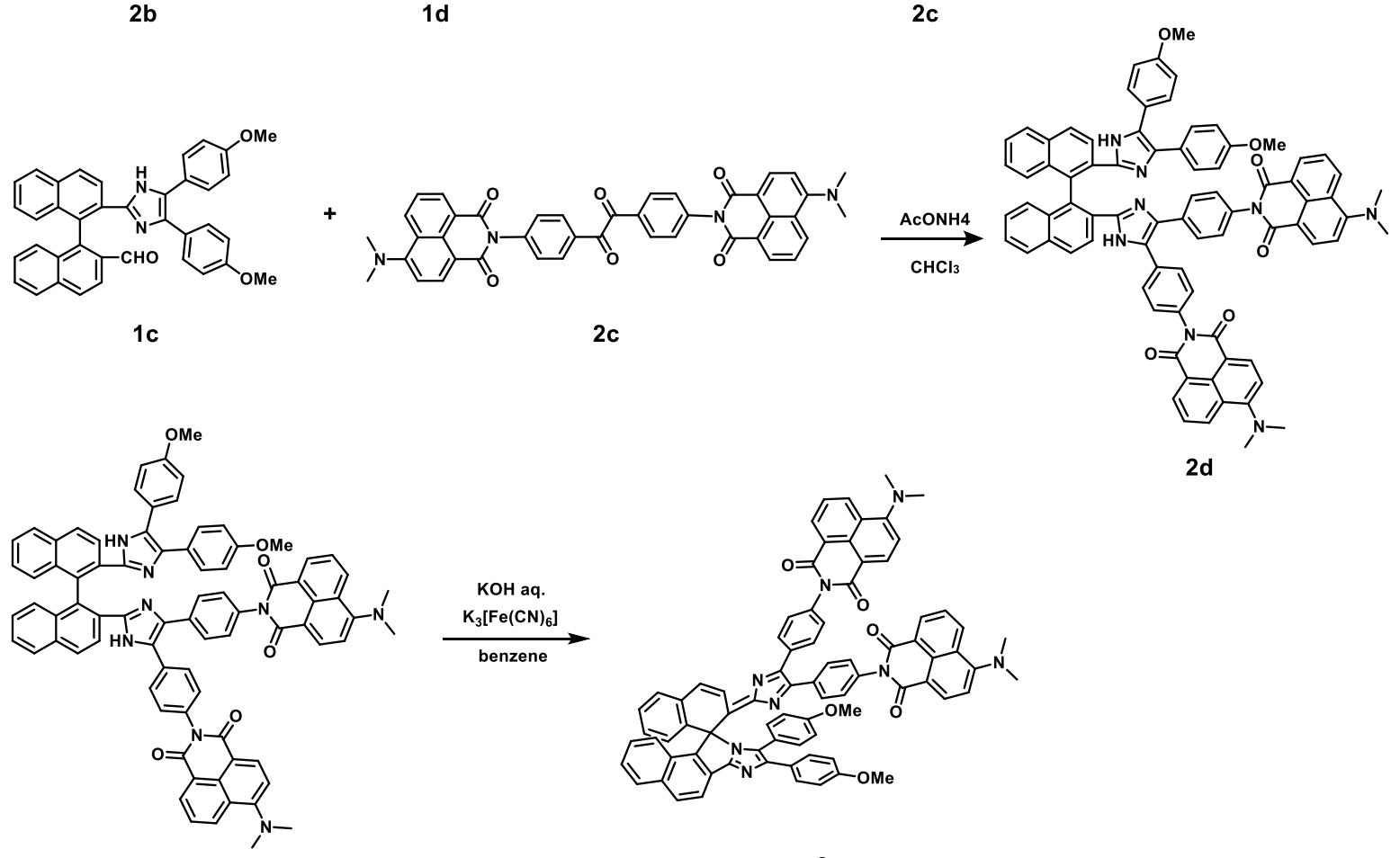

2d

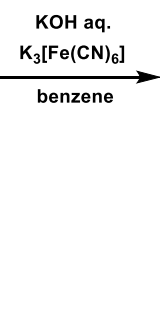

benzene

1

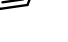

2 
4,4'-dinitrobenzil (2a) was synthesized according to a literature procedure. ${ }^{\mathrm{S} 1}$

\section{4,4'-diamino-benzil (2b)}

A mixture of 4,4'-dinitro-benzil (2a) (101 mg, $0.335 \mathrm{mmol})$, iron (290 mg, 5.20 mmol) and ammonium chloride $(39 \mathrm{mg}, 0.74 \mathrm{mmol})$ in ethanol $(8 \mathrm{~mL})$ and water $(3 \mathrm{~mL})$ was heated under reflux for 25 hours. The reaction mixture was cooled to room temperature and filtered through a celite pad. The filtrate was added water and extracted with AcOEt. The

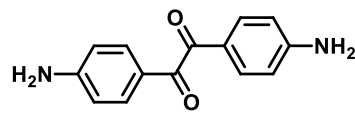

2b organic extract was washed with water and brine, and passed through a phase separator paper. After removal of the solvents, the crude product was purified by silica gel column chromatography ( $\left.\mathrm{AcOEt} / \mathrm{CH}_{2} \mathrm{Cl}_{2}=1 / 2\right)$, to give the desired product as an orange solid (41 mg, 50 \%). ${ }^{1} \mathrm{H}$ NMR (400 MHz, DMSO-d $\left.)\right) \delta: 7.50$ (d, $\left.J=8.7 \mathrm{~Hz}, 4 \mathrm{H}\right), 6.59$ (d, $\left.J=8.8 \mathrm{~Hz}, 4 \mathrm{H}\right), 6.44$ (s, 4H); HRMS (ESI-TOF) calculated for $\mathrm{C}_{14} \mathrm{H}_{12} \mathrm{~N}_{2} \mathrm{O}_{2}[\mathrm{M}+\mathrm{Na}]^{+}:$263.0791, found: 263.0780 .

\section{2,2'-(oxalylbis(4,1-phenylene))bis(6-(dimethylamino)-1H-benzo[de]isoquinoline-1,3(2H)-dione) (2c)}

4,4'-diamino-benzil (2b) (55 $\mathrm{mg}, \quad 0.23 \quad \mathrm{mmol}), \quad 4-$ (dimethylamino)-1,8-naphthalic anhydride (1d) (164 mg, $0.681 \mathrm{mmol})$ and zinc acetate $(90 \mathrm{mg}, 0.49 \mathrm{mmol})$ were dissolved in pyridine $(7 \mathrm{~mL})$. The solution was refluxed for 54 hour at $120{ }^{\circ} \mathrm{C}$. The resulting mixture was cooled to room temperature and neutralized with aqueous $1 \mathrm{~N} \mathrm{HCl}$

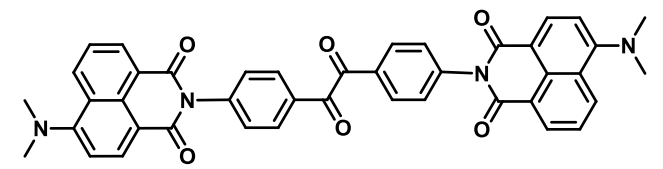

2c aq. and filtered. The filtrate was extracted with $\mathrm{CH}_{2} \mathrm{Cl}_{2}$ and washed with water and brine, and passed through a phase separator paper. After removal of the solvents, the crude product was purified by silica gel column chromatography $\left(\mathrm{AcOEt} / \mathrm{CH}_{2} \mathrm{Cl}_{2}=1 / 10\right)$ and preparative thin layer chromatography $\left(\mathrm{AcOEt} / \mathrm{CH}_{2} \mathrm{Cl}_{2}=1 / 8\right)$, to give the desired product as a pale yellow solid $(31 \mathrm{mg}, 20 \%)$. ${ }^{1} \mathrm{H}$ NMR $\left(400 \mathrm{MHz}, \mathrm{DMSO}-d_{6}\right) \delta: 8.60(\mathrm{~d}, J=8.6 \mathrm{~Hz}, 2 \mathrm{H}), 8.50(\mathrm{~d}, J=6.7 \mathrm{~Hz}, 2 \mathrm{H}), 8.38(\mathrm{~d}, J=8.3 \mathrm{~Hz}, 2 \mathrm{H}), 8.17(\mathrm{~d}, J=8.4 \mathrm{~Hz}$, 2H), $7.81(\mathrm{t}, J=7.9 \mathrm{~Hz}, 2 \mathrm{H}), 7.69$ (d, $J=8.5 \mathrm{~Hz}, 2 \mathrm{H}), 7.27(\mathrm{~d}, J=8.4 \mathrm{~Hz}, 2 \mathrm{H}), 3.14(\mathrm{~s}, 12 \mathrm{H}) .{ }^{13} \mathrm{C} \mathrm{NMR}\left(100 \mathrm{MHz}, \mathrm{CDCl}_{3}\right) \delta$ : $198.90,168.95,168.24,162.15,148.01,137.69,137.21,137.09,136.01,135.85,135.63,135.51,130.24,129.56,127.91,118.62$, 118.19, 49.65; HRMS (ESI-TOF) calculated for $\mathrm{C}_{42} \mathrm{H}_{30} \mathrm{~N}_{4} \mathrm{O}_{6}[\mathrm{M}+\mathrm{Na}]^{+}:$709.2058, found: 709.2058.

\section{2,2'-((2-(2'-(4,5-bis(4-methoxyphenyl)-1H-imidazol-2-yl)-[1,1'-binaphthalen]-2-yl)-1H-imidazole-4,5-diyl)bis(4,1-}

\section{phenylene))bis(6-(dimethylamino)-1H-benzo[de $]$ isoquinoline-1,3(2H)-dione)}

(2d)

A mixture of 2'-(4,5-bis(4-methoxyphenyl)-1H-imidazol-2-yl)-[1,1'binaphthalene]-2-carbaldehyde (1c) (42 mg, $0.074 \mathrm{mmol}), 2,2$-(oxalylbis(4,1phenylene)) bis(6-(dimethylamino)-1H-benzo[de]isoquinoline-1,3(2H)-dione) (2c) $(33 \mathrm{mg}, 0.048 \mathrm{mmol})$ and ammonium acetate $(198 \mathrm{mg}, 2.56 \mathrm{mmol})$ in $\mathrm{CHCl}_{3}(3 \mathrm{~mL})$ was stirred at $110^{\circ} \mathrm{C}$ for 67 hours in a sealed tube. After cooling to room temperature, the reaction mixture was added water and extracted with $\mathrm{CHCl}_{3}$. The organic extract was washed with water and brine, and passed through a phase separator paper. The product was purified by silica gel column chromatography $\left(\mathrm{AcOEt} / \mathrm{CH}_{2} \mathrm{Cl}_{2}=1 / 3\right)$ to give desired product as a light yellow solid (38 mg, $64 \%$ ). ${ }^{1} \mathrm{H}$ NMR (400 MHz,

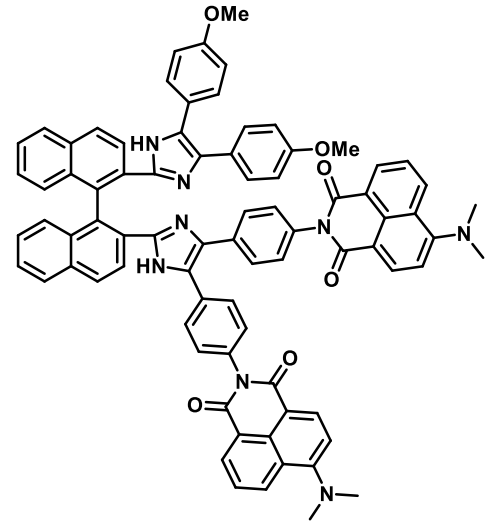

2d 
DMSO-d6) $\delta: 14.43(\mathrm{~s}, 1 \mathrm{H}), 13.53(\mathrm{~s}, 1 \mathrm{H}), 8.55(\mathrm{~d}, J=8.5 \mathrm{~Hz}, 2 \mathrm{H}), 8.44(\mathrm{~d}, J=6.8 \mathrm{~Hz}, 2 \mathrm{H}), 8.32(\mathrm{dd}, J=8.2 \mathrm{~Hz}, J=3.6 \mathrm{~Hz}, 2 \mathrm{H})$, $8.21(\mathrm{t}, J=8.0 \mathrm{~Hz}, 2 \mathrm{H}), 8.09-8.04(\mathrm{~m}, 4 \mathrm{H}), 7.78(\mathrm{t}, J=7.9 \mathrm{~Hz}, 2 \mathrm{H}), 7.50(\mathrm{t}, J=7.6 \mathrm{~Hz}, 2 \mathrm{H}), 8.16(\mathrm{~d}, J=8.2 \mathrm{~Hz}, 2 \mathrm{H}), 7.37-7.12$ (m, 14H), 6.98-6.95 (m, 2H), 6.88 (d, $J=8.6 \mathrm{~Hz}, 2 \mathrm{H}), 6.77$ (d, J=8.7 Hz, 2H), 3.73 (s, 3H), 3.69 (s, 3H), 3.11 (s, 12H); HRMS (ESI-TOF) calculated for $\mathrm{C}_{80} \mathrm{H}_{58} \mathrm{~N}_{8} \mathrm{O}_{6}[\mathrm{M}+\mathrm{H}]^{+}:$1227.4552, found: 1227.4514 .

\section{Compound 2}

All manipulations were carried out with the exclusion of light. Under nitrogen, to a solution of 2,2'-((2-(2'-(4,5-bis(4-methoxyphenyl)-1H-imidazol-2-yl)- [1,1'binaphthalen]-2-yl)-1H-imidazole-4,5-diyl)bis(4,1-phenylene))bis(6-

(dimethylamino)-1H-benzo[de]isoquinoline-1,3(2H)-dione) (2d) (39 $\mathrm{mg}, 0.032$ $\mathrm{mmol})$ in benzene $(16 \mathrm{~mL}$ ) was added a solution of potassium ferricyanide $(946 \mathrm{mg}$, $2.87 \mathrm{mmol})$ and $\mathrm{KOH}(375 \mathrm{mg}, 6.68 \mathrm{mmol})$ in water $(12 \mathrm{~mL})$, and the reaction mixture was vigorously stirred for 4 hours. The organic layer was separated, washed with water, and concentrated. The crude product was purified with silica gel column chromatography $\left(\mathrm{CH}_{2} \mathrm{Cl}_{2} / \mathrm{AcOEt}=5 / 1\right)$ and reverse phase HPLC $\left(\mathrm{CH}_{3} \mathrm{CN}\right)$ to yield

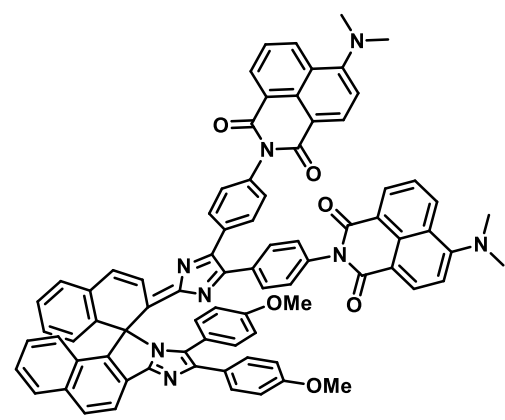

2 the desired product as a dark red solid (15 mg, $38 \%) .{ }^{1} \mathrm{H}$ NMR (400 MHz, DMSO- $\left.d_{6}\right) \delta: 8.55(\mathrm{~d}, J=8.5 \mathrm{~Hz}, 2 \mathrm{H}), 8.46(\mathrm{dd}, J=$ $14.7 \mathrm{~Hz}, J=6.6 \mathrm{~Hz}, 2 \mathrm{H}), 8.34(\mathrm{dd}, J=15.3 \mathrm{~Hz}, J=6.6 \mathrm{~Hz}, 2 \mathrm{H}), 8.22(\mathrm{~d}, J=8.4 \mathrm{~Hz}, 1 \mathrm{H}), 8.12(\mathrm{~d}, J=8.6 \mathrm{~Hz}, 1 \mathrm{H}), 8.00(\mathrm{~d}, J=7.7$ $\mathrm{Hz}, 1 \mathrm{H}), 7.88$ (d, $J=9.8 \mathrm{~Hz}, 1 \mathrm{H}), 7.80-7.75(\mathrm{~m}, 2 \mathrm{H}), 7.69$ (d, $J=8.5 \mathrm{~Hz}, 2 \mathrm{H}), 7.52-7.48(\mathrm{~m}, 3 \mathrm{H}), 7.41-7.37$ (m, 6H), 7.26-7.20 (m, 6H), 7.10 (d, $J=8.4 \mathrm{~Hz}, 2 \mathrm{H}), 6.97-6.92(\mathrm{~m}, 4 \mathrm{H}), 6.89$ (d, $J=7.9 \mathrm{~Hz}, 1 \mathrm{H}), 6.61(\mathrm{~d}, J=8.3 \mathrm{~Hz}, 2 \mathrm{H}), 3.81(\mathrm{~s}, 3 \mathrm{H}), 3.76$ (s, 3H), $3.11(\mathrm{~d}, J=1.0 \mathrm{~Hz}, 12 \mathrm{H}) .{ }^{13} \mathrm{C} \mathrm{NMR}\left(100 \mathrm{MHz}, \mathrm{CDCl}_{3}\right) \delta: 164.67,164.47,164.06,161.37,160.55,159.12,127.12,137.51,132.87$, $132.29,131.71,131.49,131.42,131.38,131.23,130.99,130.91,130.38,129.88,129.47,129.16,128.48,128.28,127.79,127.68$, 127.09, 126.30, 126.18, 125.67, 125.30, 124.92, 124.88, 122,94, 122.84, 118.18, 114.38, 114.30, 114.21, 113.65, 113.55, 113.48, 113.35, 66.29, 64.72, 55.30, 55.24, 44.79, 38.92, 38.78; HRMS (ESI-TOF) calculated for $\mathrm{C}_{80} \mathrm{H}_{56} \mathrm{~N}_{8} \mathrm{O}_{6}[\mathrm{M}+\mathrm{Na}]^{+}:$: 247.4215 , found: 1247.4227. 


\section{2. ${ }^{1}$ H NMR Spectra}

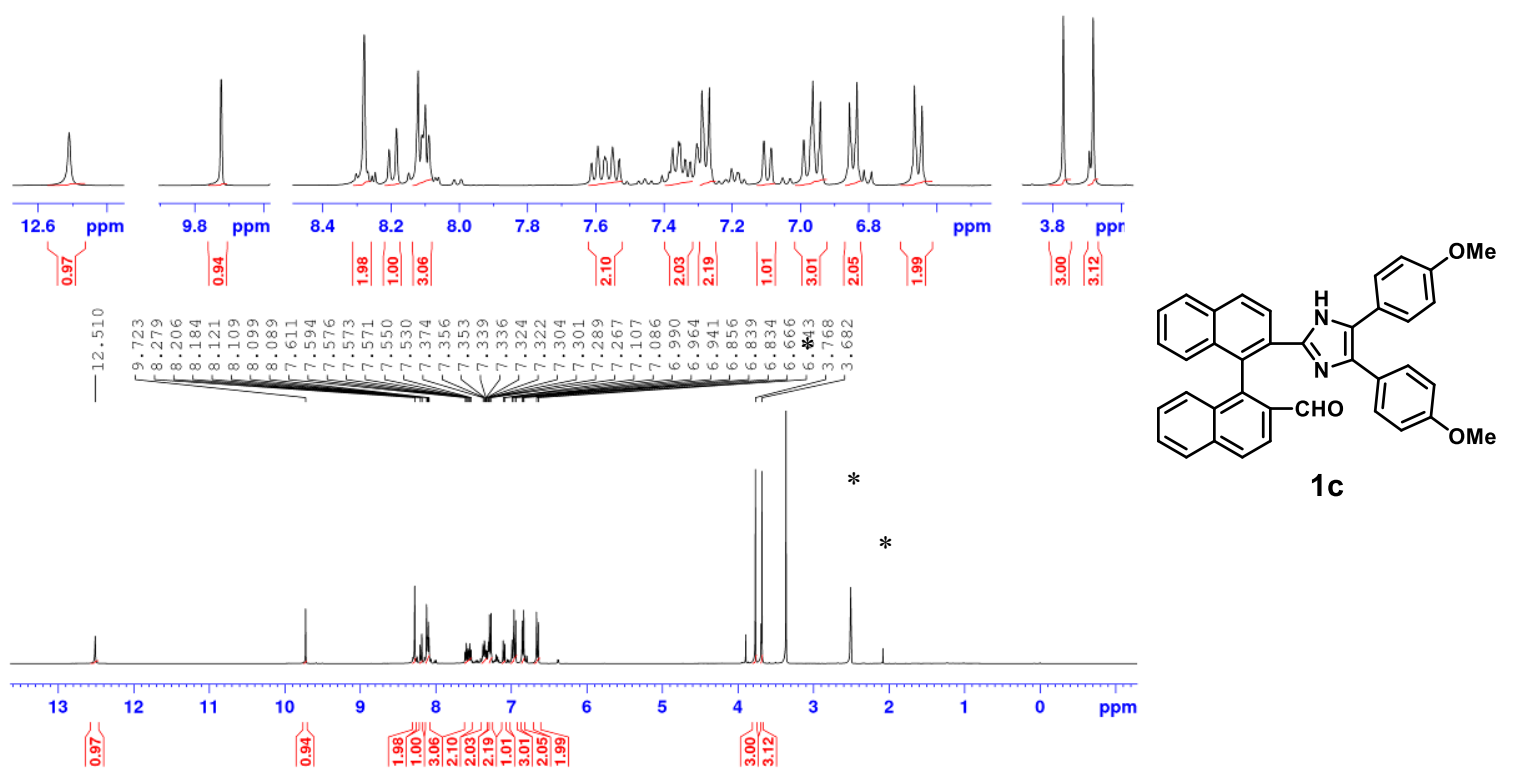

Figure S3. ${ }^{1} \mathrm{H}$ NMR spectrum of 2'-(4,5-bis(4-methoxyphenyl)-1H-imidazol-2-yl)-[1,1'-binaphthalene]-2-carbaldehyde (1c) in DMSO- $d_{6}$ (* solvent peaks).

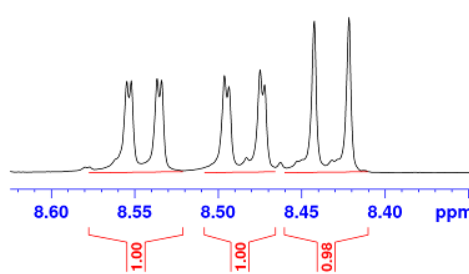

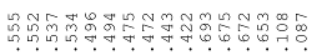
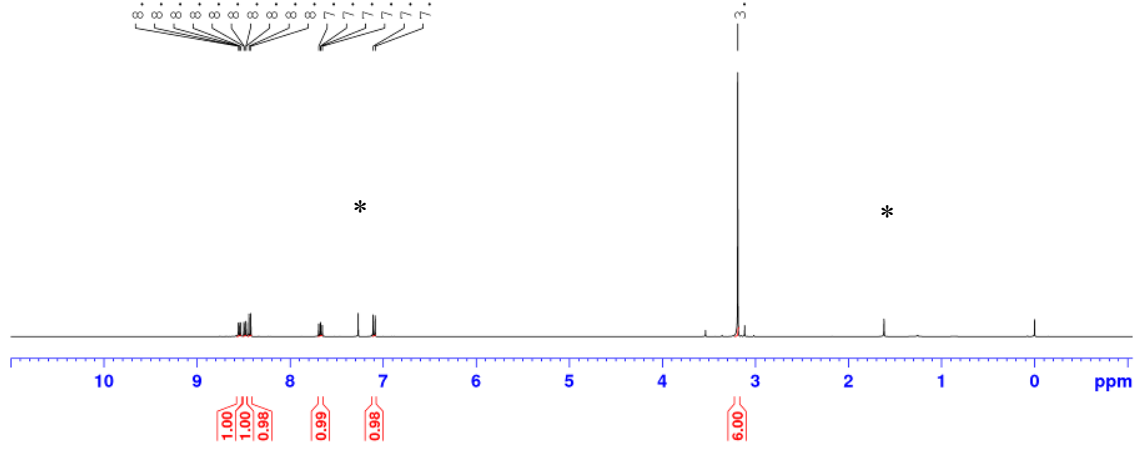<smiles>CN(C)c1ccc2c3c(cccc13)C(=O)OC2=O</smiles>

$1 d$ 

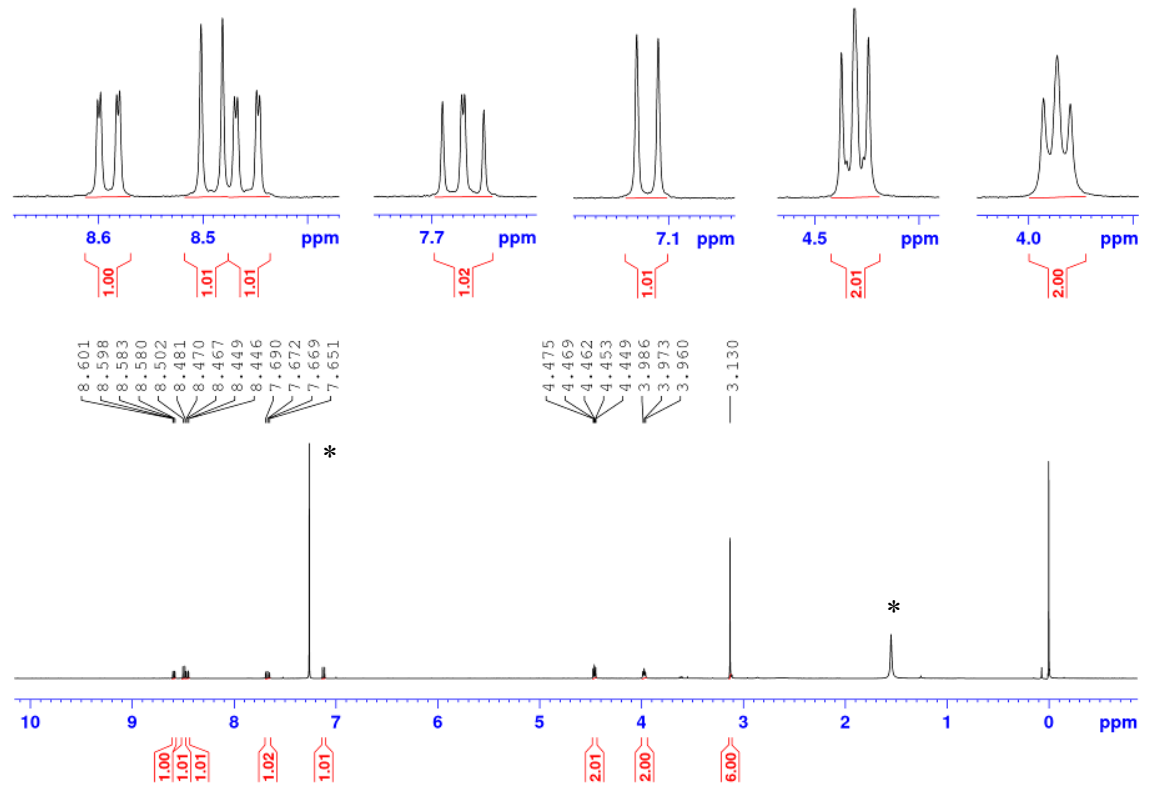

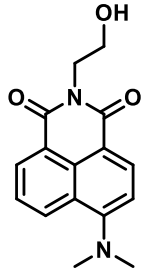

1e

Figure S5. ${ }^{1} \mathrm{H}$ NMR spectrum of 6-(dimethylamino)-2-(2-hydroxyethyl)-1H-benzo[de]isoquinoline-1,3(2H)-dione (1e) in $\mathrm{CDCl}_{3}$ (* solvent peaks).
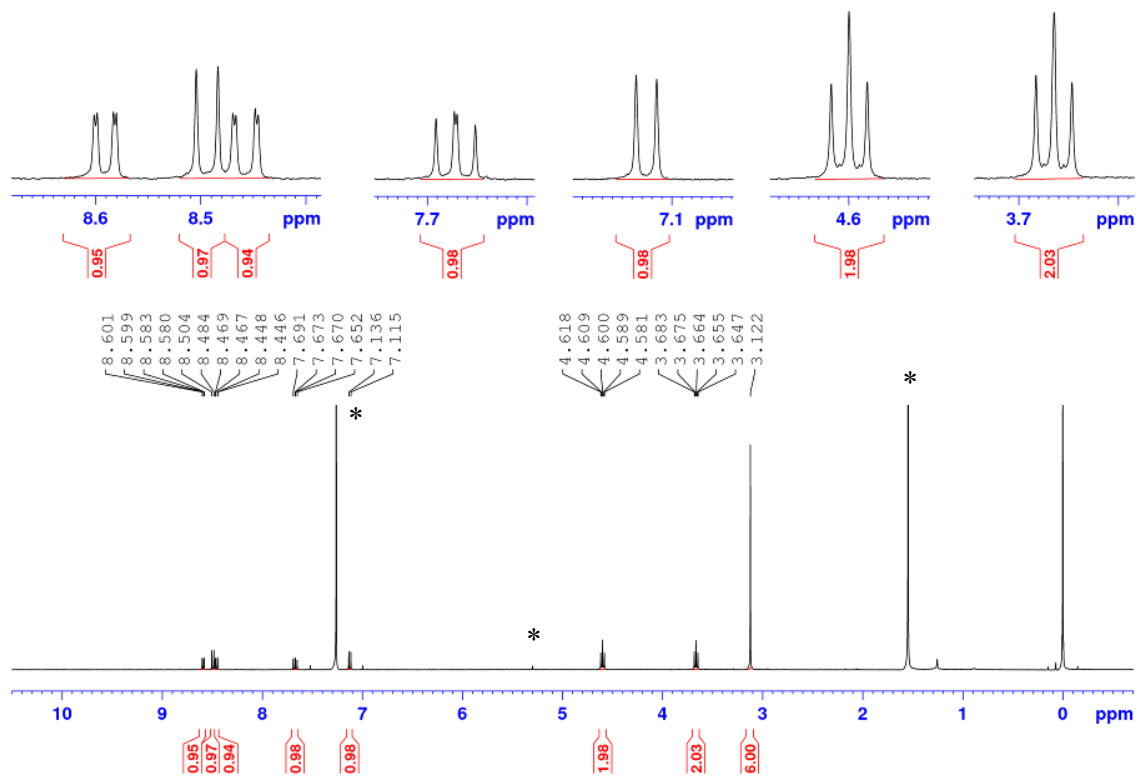

Figure S6. ${ }^{1} \mathrm{H}$ NMR spectrum of 2-(2-bromoethyl)-6-(dimethylamino)-1H-benzo[de]isoquinoline-1,3(2H)-dione (1f) in $\mathrm{CDCl}_{3}(*$ solvent peaks). 

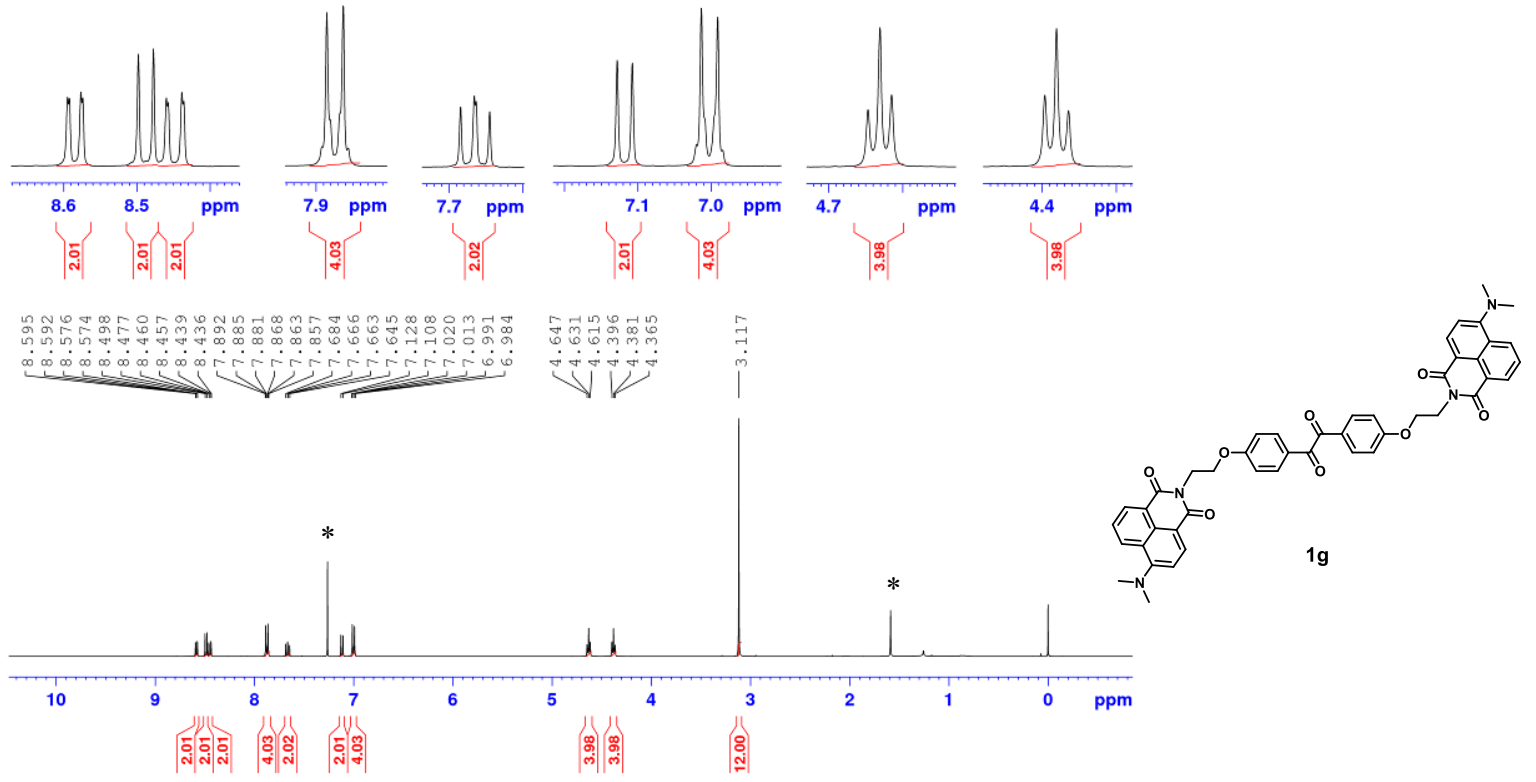

Figure S7. ${ }^{1} \mathrm{H}$ NMR spectrum of 2,2'-(((oxalylbis(4,1-phenylene))bis(oxy))bis(ethane-2,1-diyl))bis(6-(dimethylamino)$1 \boldsymbol{H}$-benzo[de]isoquinoline-1,3(2H)-dione) (1g) in $\mathrm{CDCl}_{3}(*$ solvent peaks).
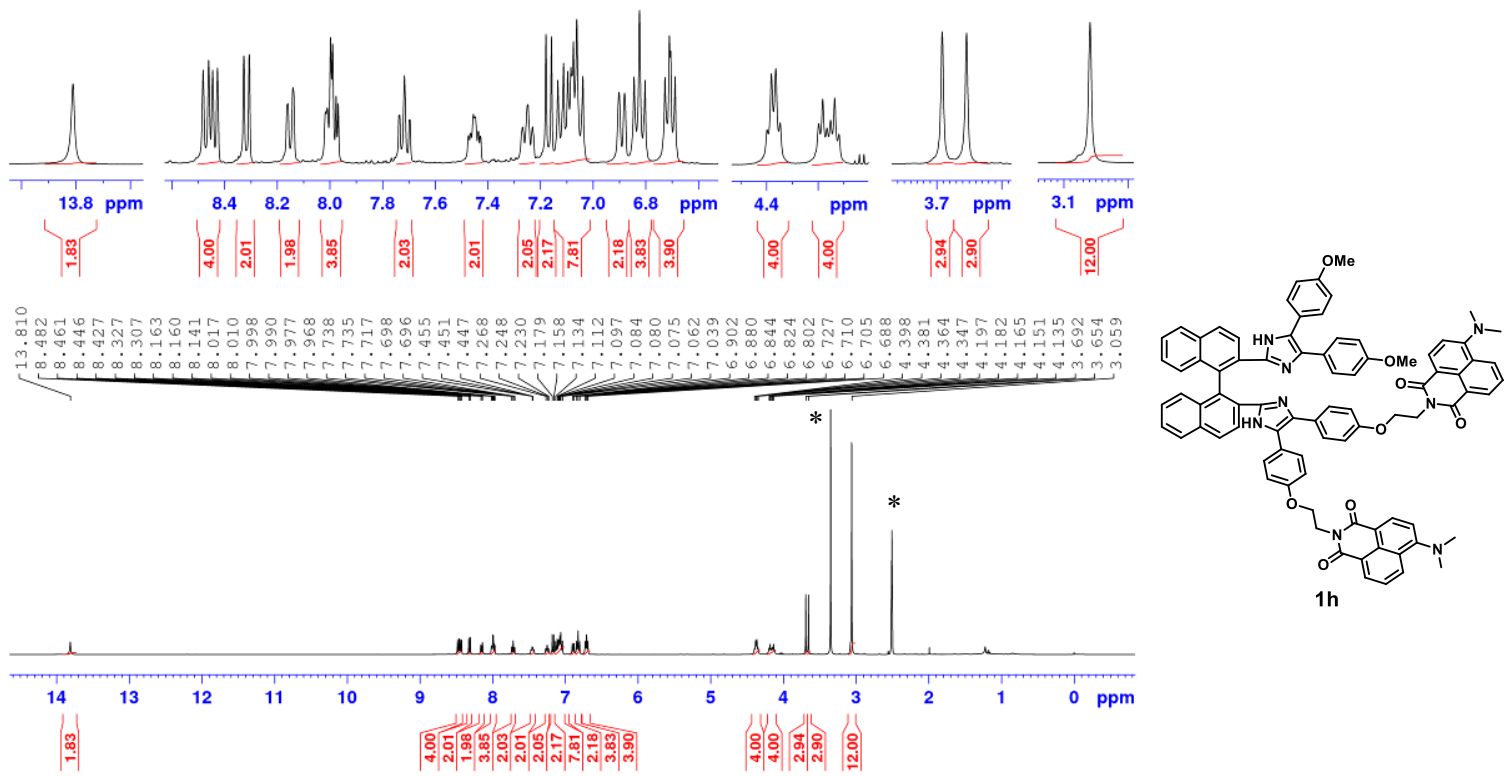

$1 \mathrm{~h}$

Figure S8. ${ }^{1} \mathrm{H}$ NMR spectrum of 2,2'-(((2-(2'-(4,5-bis(4-methoxyphenyl)-1H-imidazol-2-yl)-[1,1'-binaphthalen]-2-yl)$1 H$-imidazole-4,5-diyl)bis(4,1-phenylene))bis(oxy))bis(ethane-2,1-diyl))bis(6-(dimethylamino)-1Hbenzo[de]isoquinoline-1,3(2H)-dione) (1h) in DMSO- $d_{6}$ (* solvent peaks). 


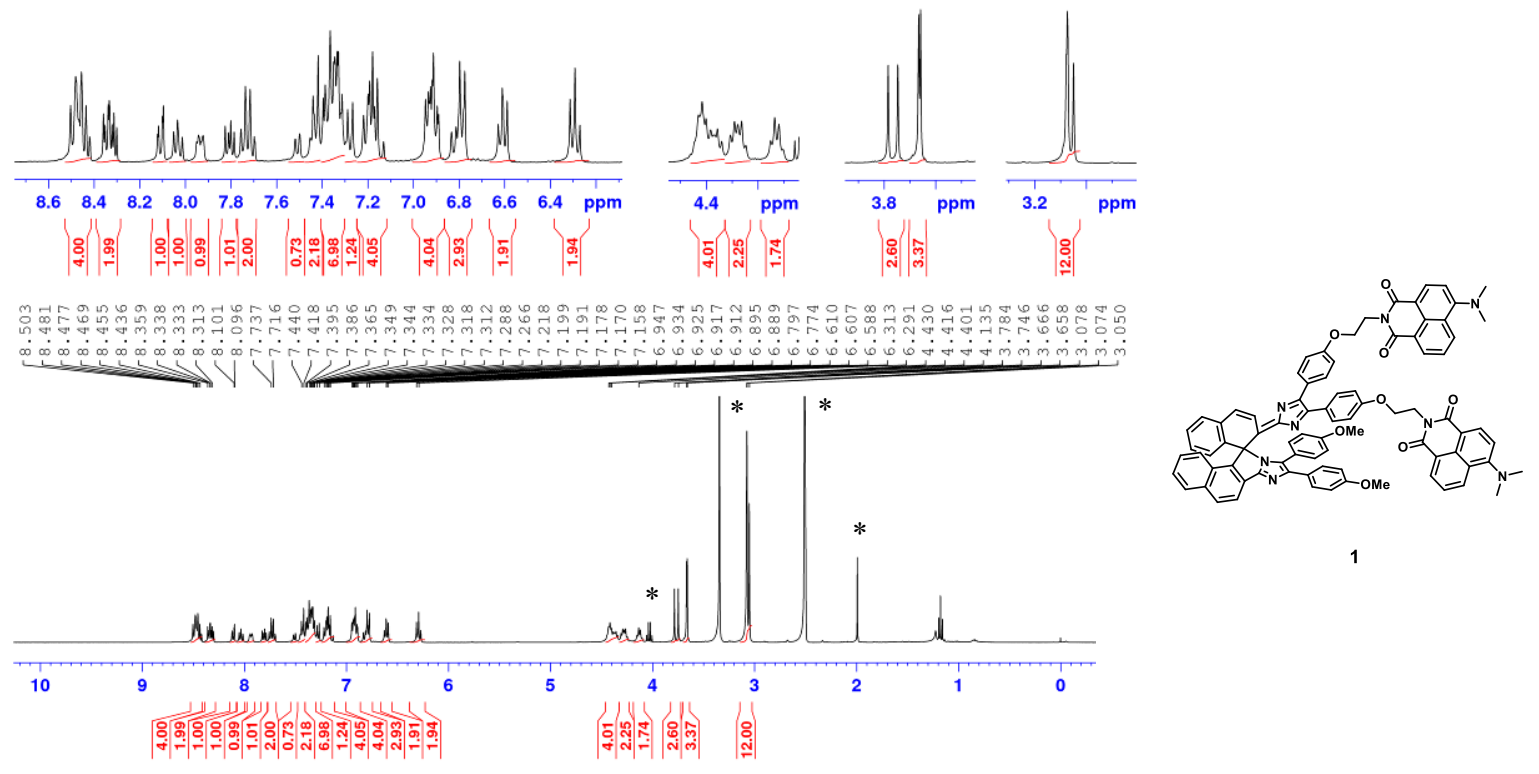

Figure S9. ${ }^{1} \mathrm{H}$ NMR spectrum of 1 in DMSO- $d_{6}$ (* solvent peaks). 


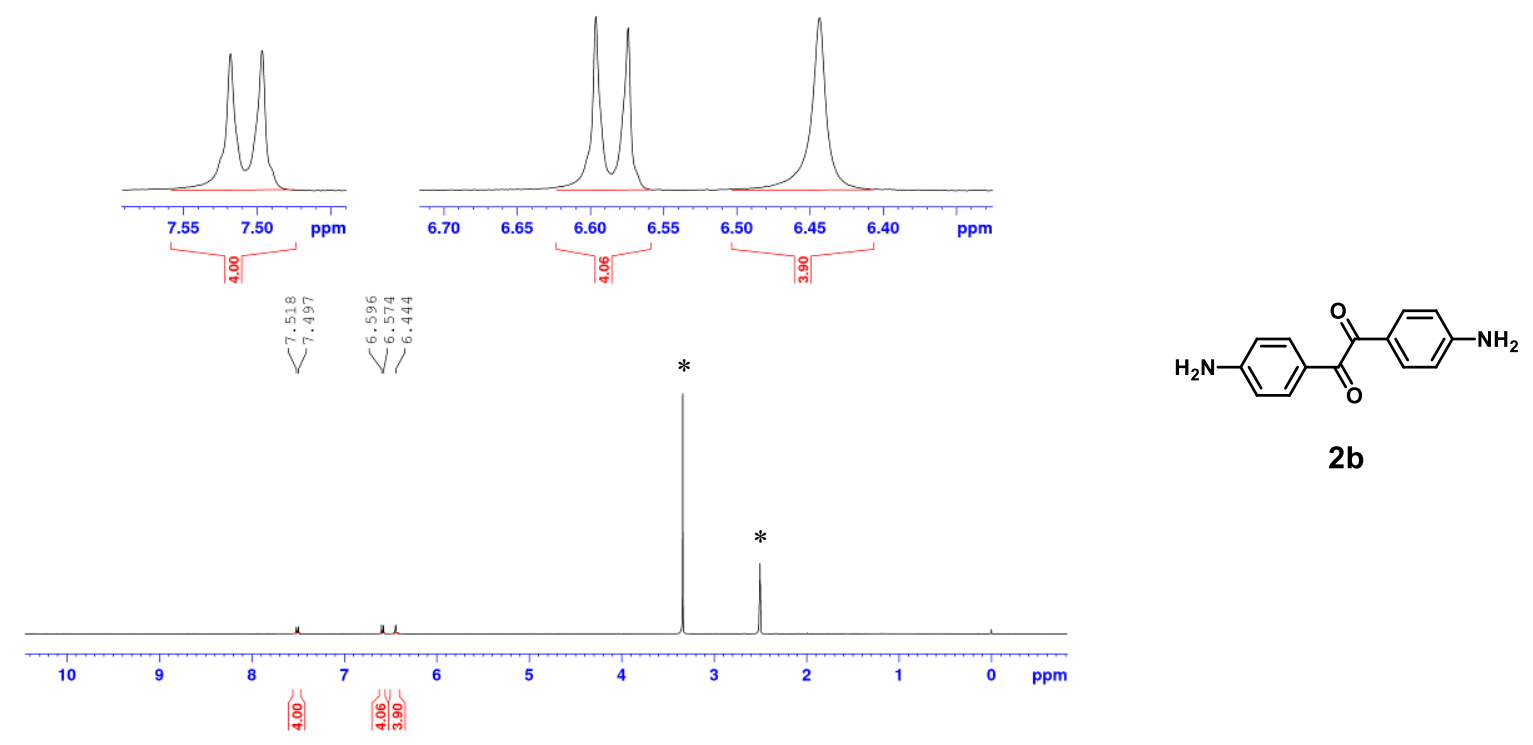

Figure S10. ${ }^{1} \mathrm{H}$ NMR spectrum of 4,4'-diamino-benzil (2b) in DMSO- $d_{6}(*$ solvent peaks).
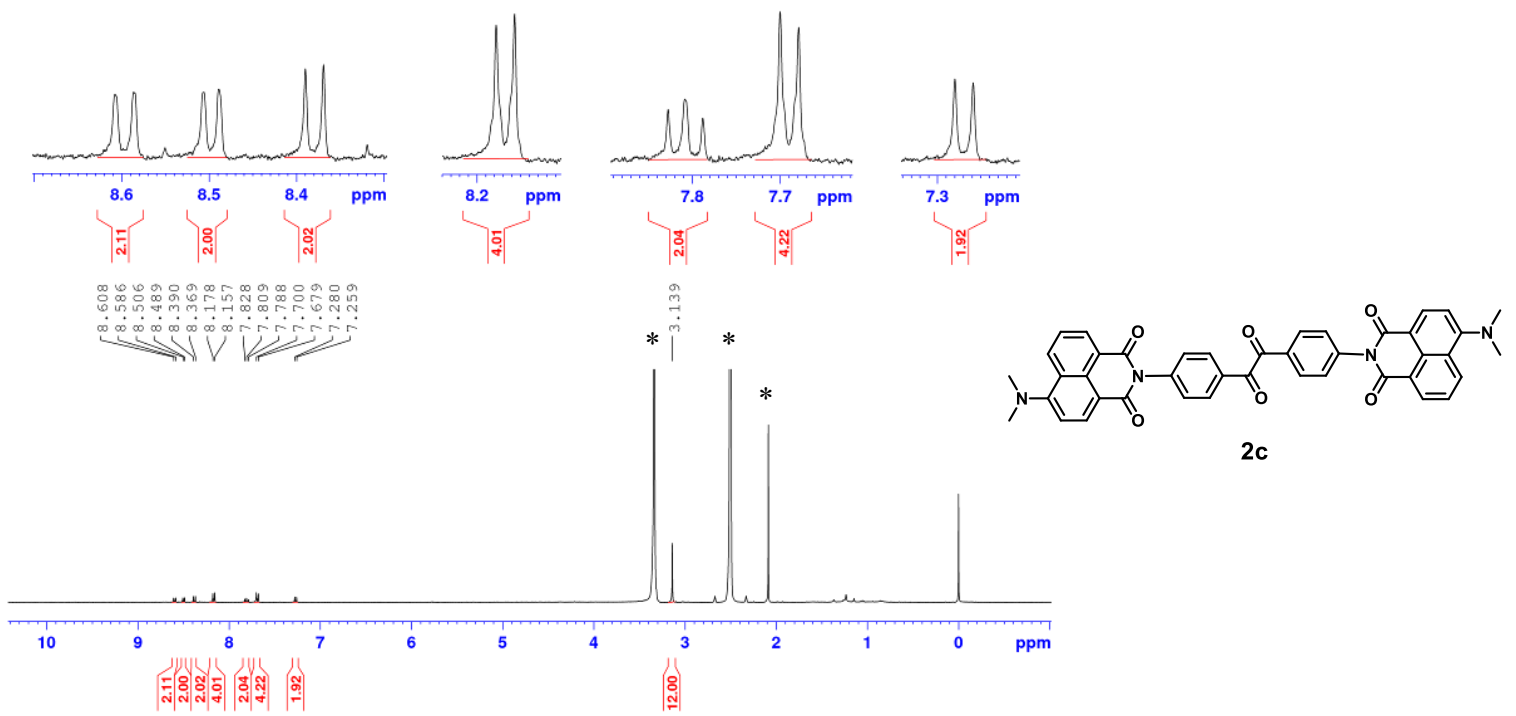

Figure S11. ${ }^{1} \mathrm{H}$ NMR spectrum of 2,2'-(oxalylbis(4,1-phenylene))bis(6-(dimethylamino)-1H-benzo[de]isoquinoline1,3(2H)-dione) (2c) in $\mathrm{CDCl}_{3}$ (* solvent peaks). 


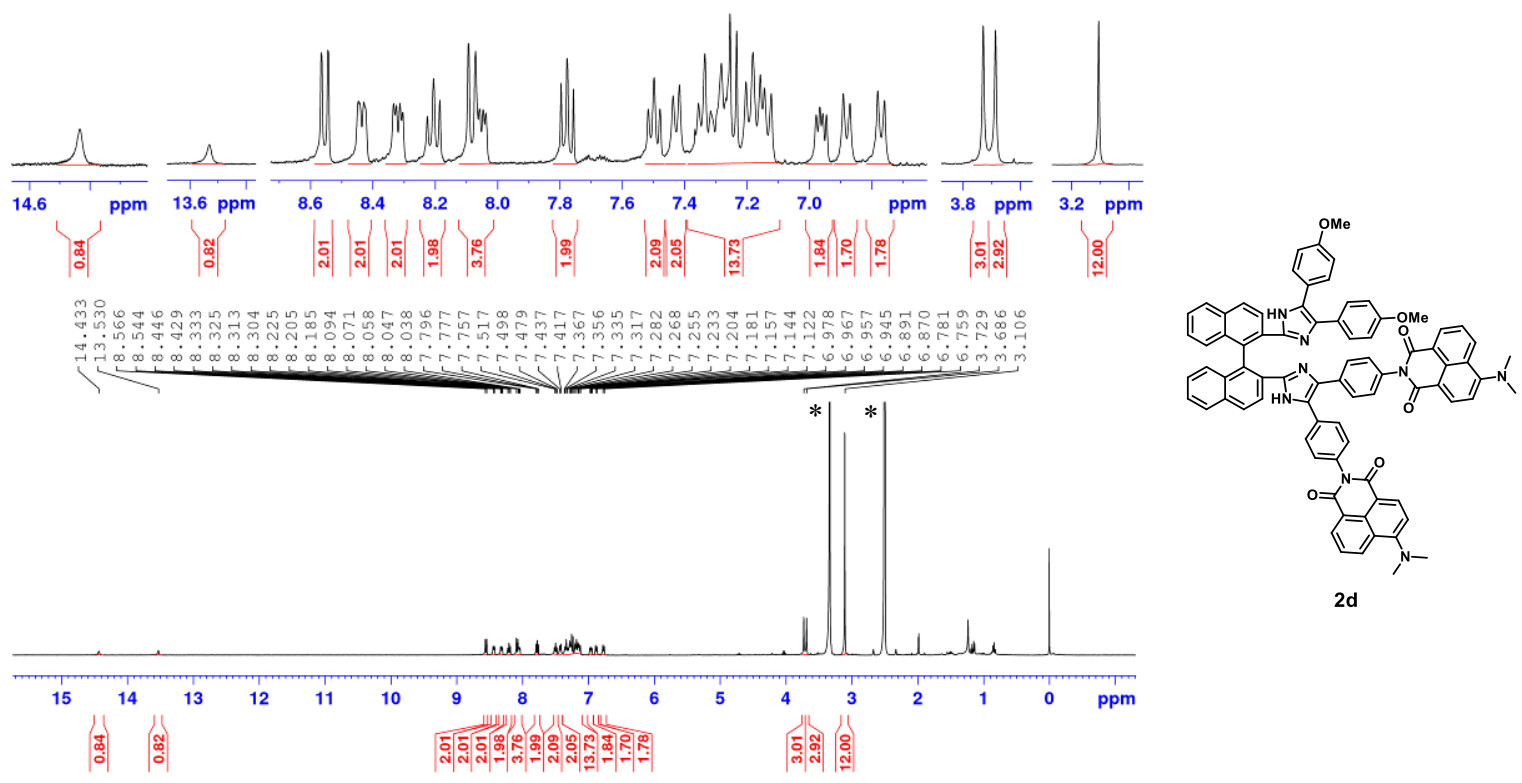

Figure S12. ${ }^{1} \mathrm{H}$ NMR spectrum of 2,2'-((2-(2'-(4,5-bis(4-methoxyphenyl)-1H-imidazol-2-yl)-[1,1'-binaphthalen]-2-yl)$1 H$-imidazole-4,5-diyl)bis(4,1-phenylene))bis(6-(dimethylamino)-1H-benzo[de $]$ isoquinoline-1,3(2H)-dione) $\quad(2 \mathrm{~d}) \quad$ in DMSO- $d_{6}(*$ solvent peaks).
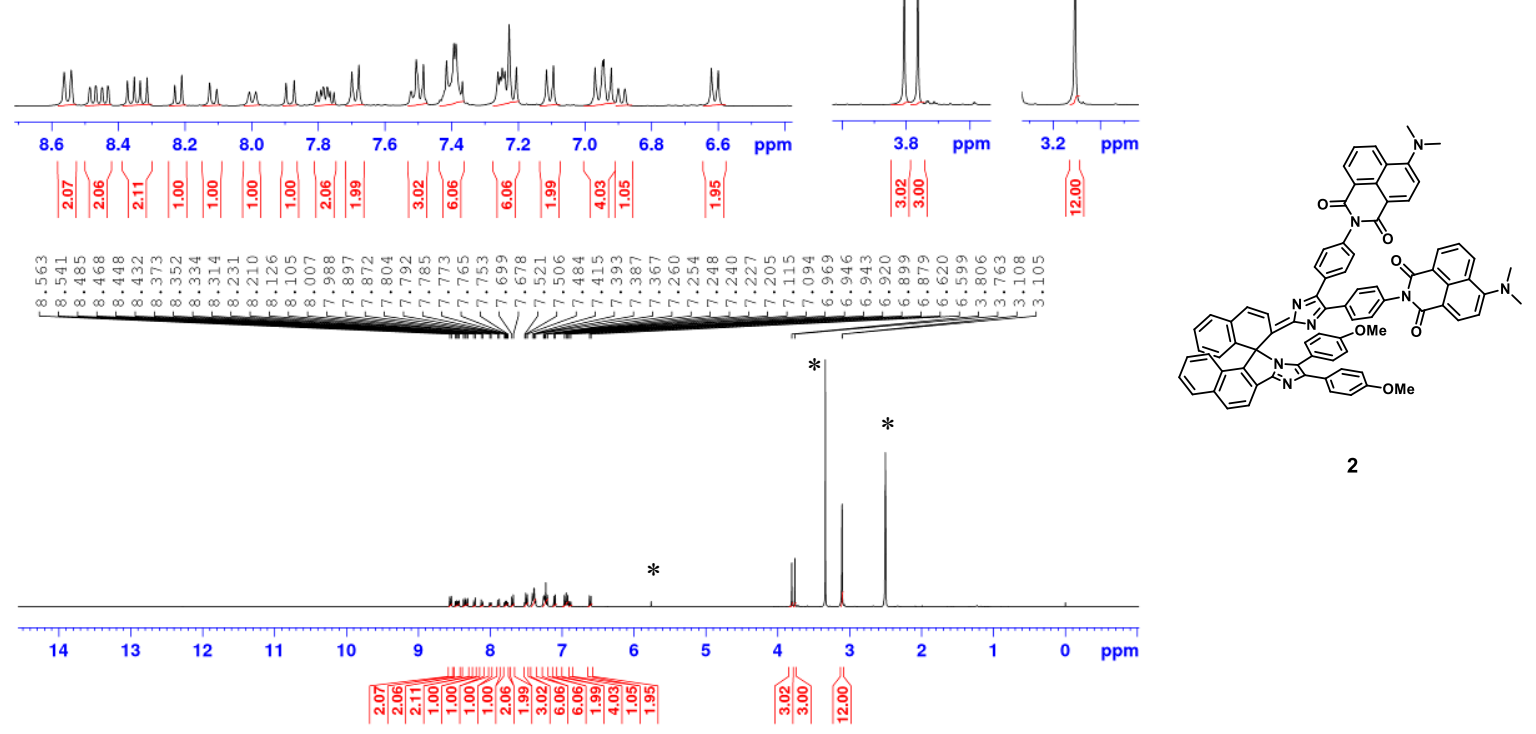

2

Figure S13. ${ }^{1} \mathrm{H}$ NMR spectrum of 2 in DMSO- $d_{6}(*$ solvent peaks). 


\section{3. ${ }^{13} \mathrm{C}$ NMR Spectra}

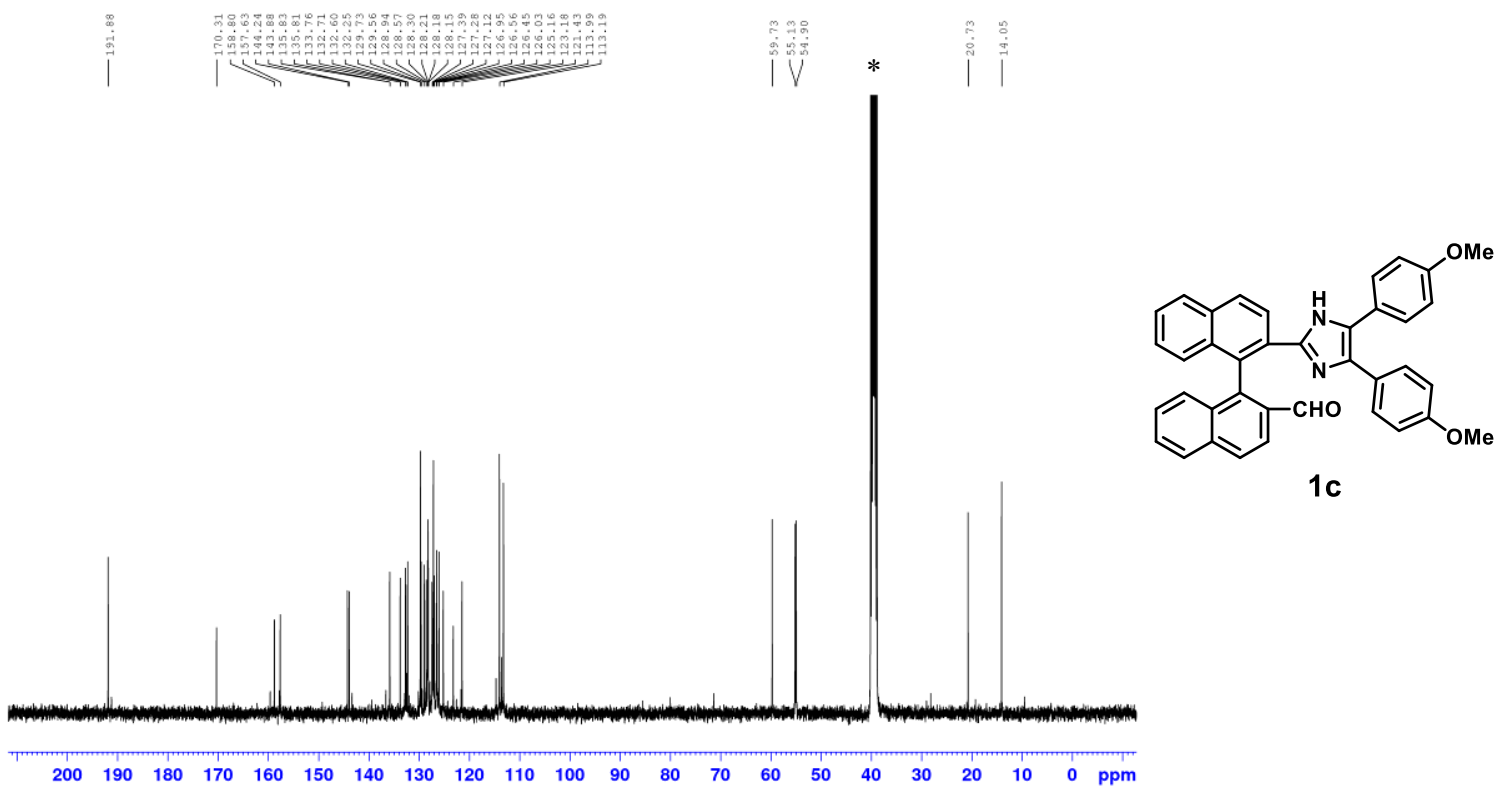

Figure S14. ${ }^{13} \mathrm{C}$ NMR spectrum of2'-(4,5-bis(4-methoxyphenyl)-1H-imidazol-2-yl)-[1,1'-binaphthalene]-2-carbaldehyde (1c) in DMSO- $d_{6}(*$ solvent peaks)

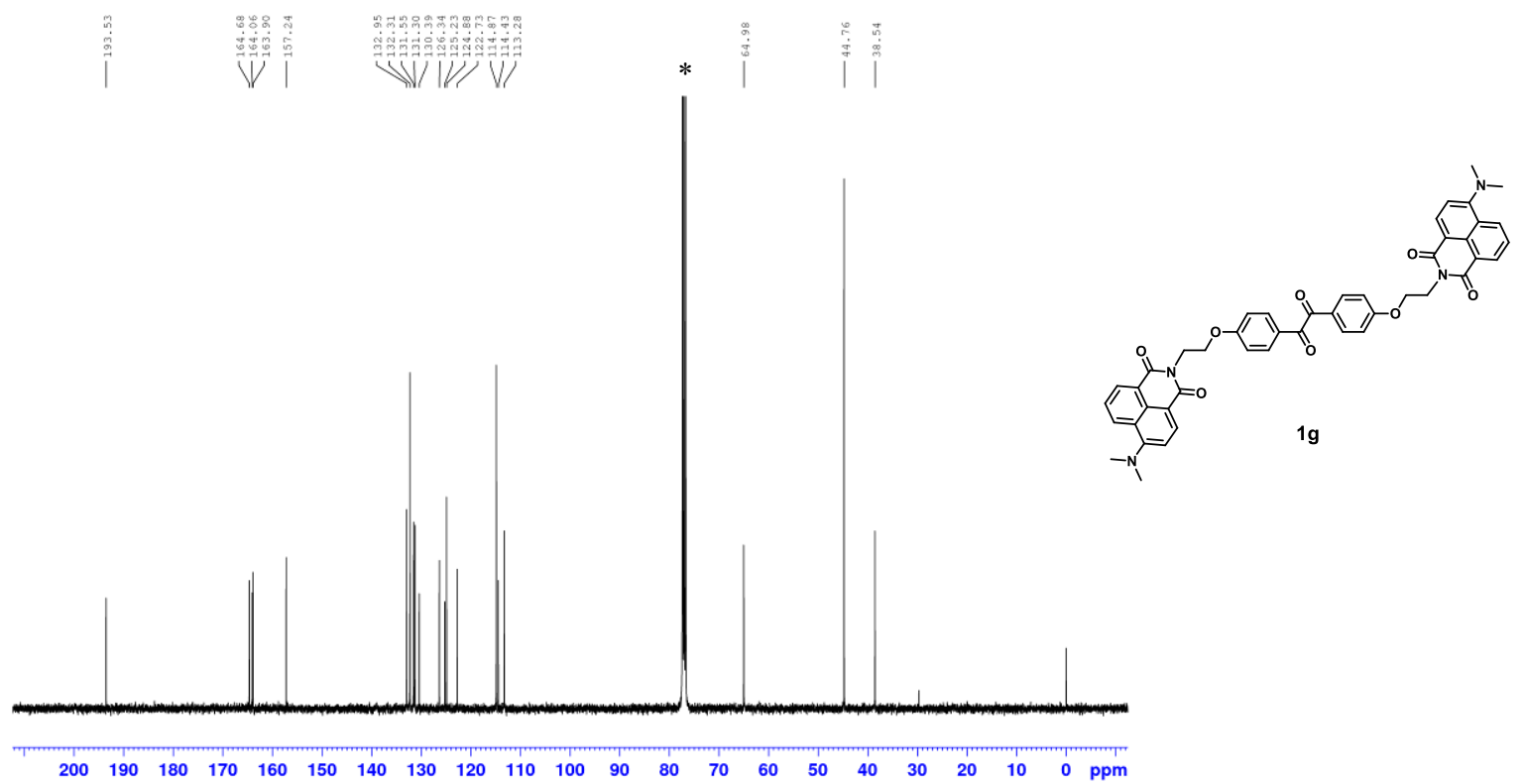

Figure S15. ${ }^{13} \mathrm{C}$ NMR spectrum of 2,2'-(((oxalylbis(4,1-phenylene))bis(oxy))bis(ethane-2,1-diyl))bis(6-(dimethylamino)-

$1 H$-benzo[de]isoquinoline-1,3(2H)-dione) (1g) in $\mathrm{CDCl}_{3}$ (* solvent peaks). 


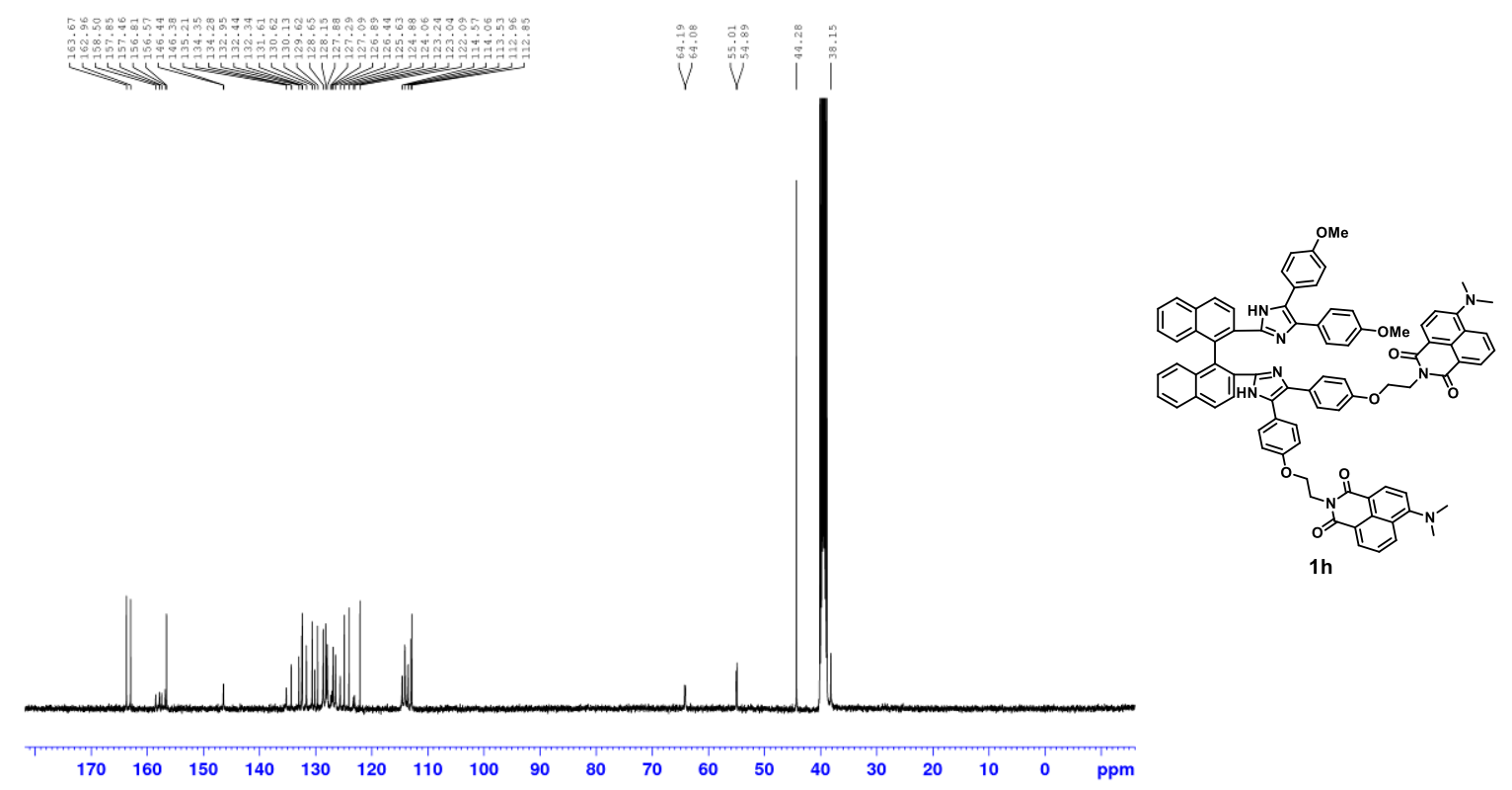

Figure S16. ${ }^{13} \mathrm{C}$ NMR spectrum of 2,2'-(((2-(2'-(4,5-bis(4-methoxyphenyl)-1H-imidazol-2-yl)-[1,1'-binaphthalen]-2-yl)$1 H$-imidazole-4,5-diyl)bis(4,1-phenylene))bis(oxy))bis(ethane-2,1-diyl))bis(6-(dimethylamino)-1Hbenzo[de] isoquinoline-1,3(2H)-dione) (1h) in DMSO- $d_{6}$ (* solvent peaks).

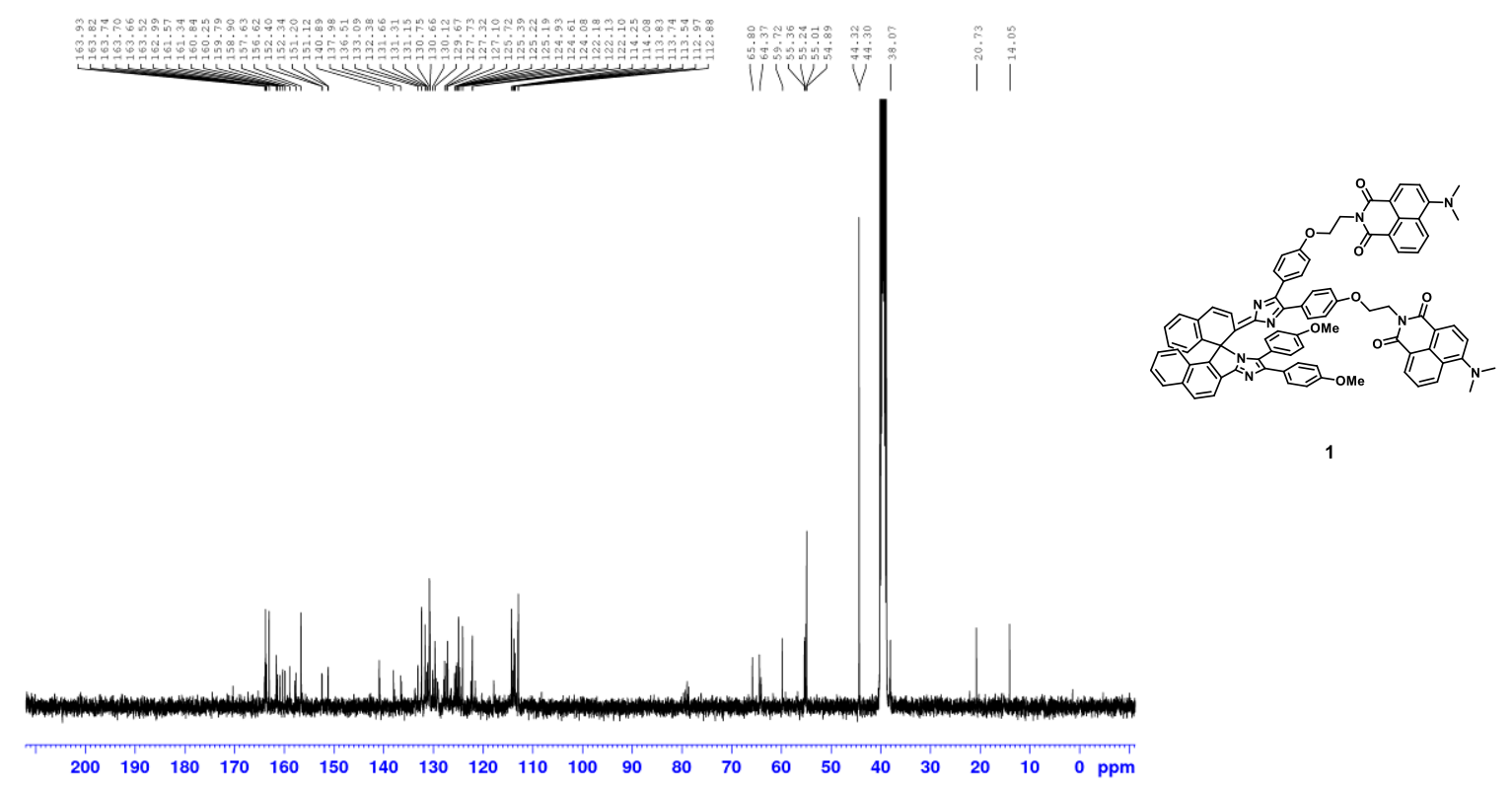

Figure S17. ${ }^{13} \mathrm{C}$ NMR spectrum of 1 in DMSO- $d_{6}(*$ solvent peaks). 


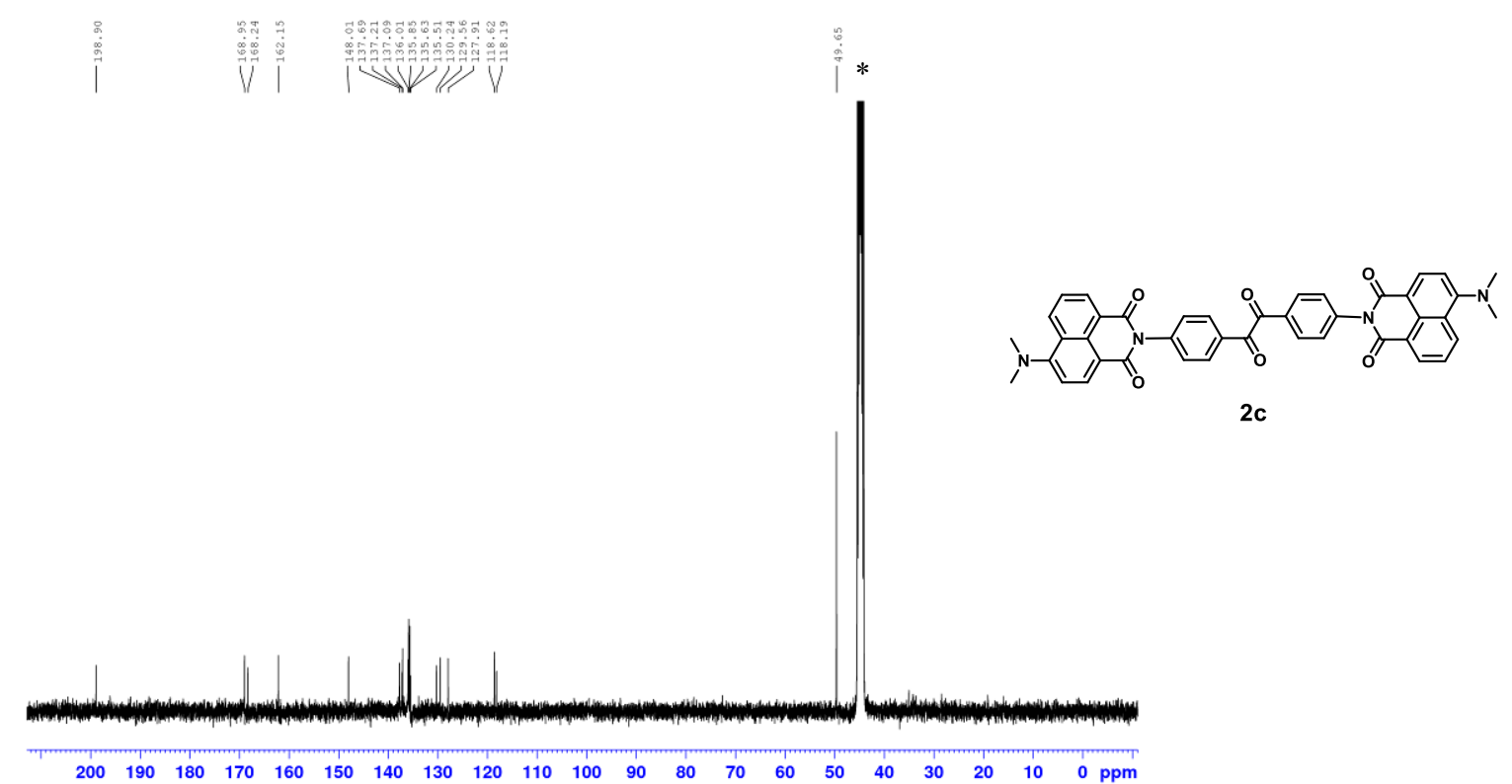

Figure S18. ${ }^{13} \mathrm{C}$ NMR spectrum of 2,2'-(oxalylbis(4,1-phenylene))bis(6-(dimethylamino)-1H-benzo[de]isoquinoline1,3(2H)-dione) (2c) in DMSO- $d_{6}(*$ solvent peaks).

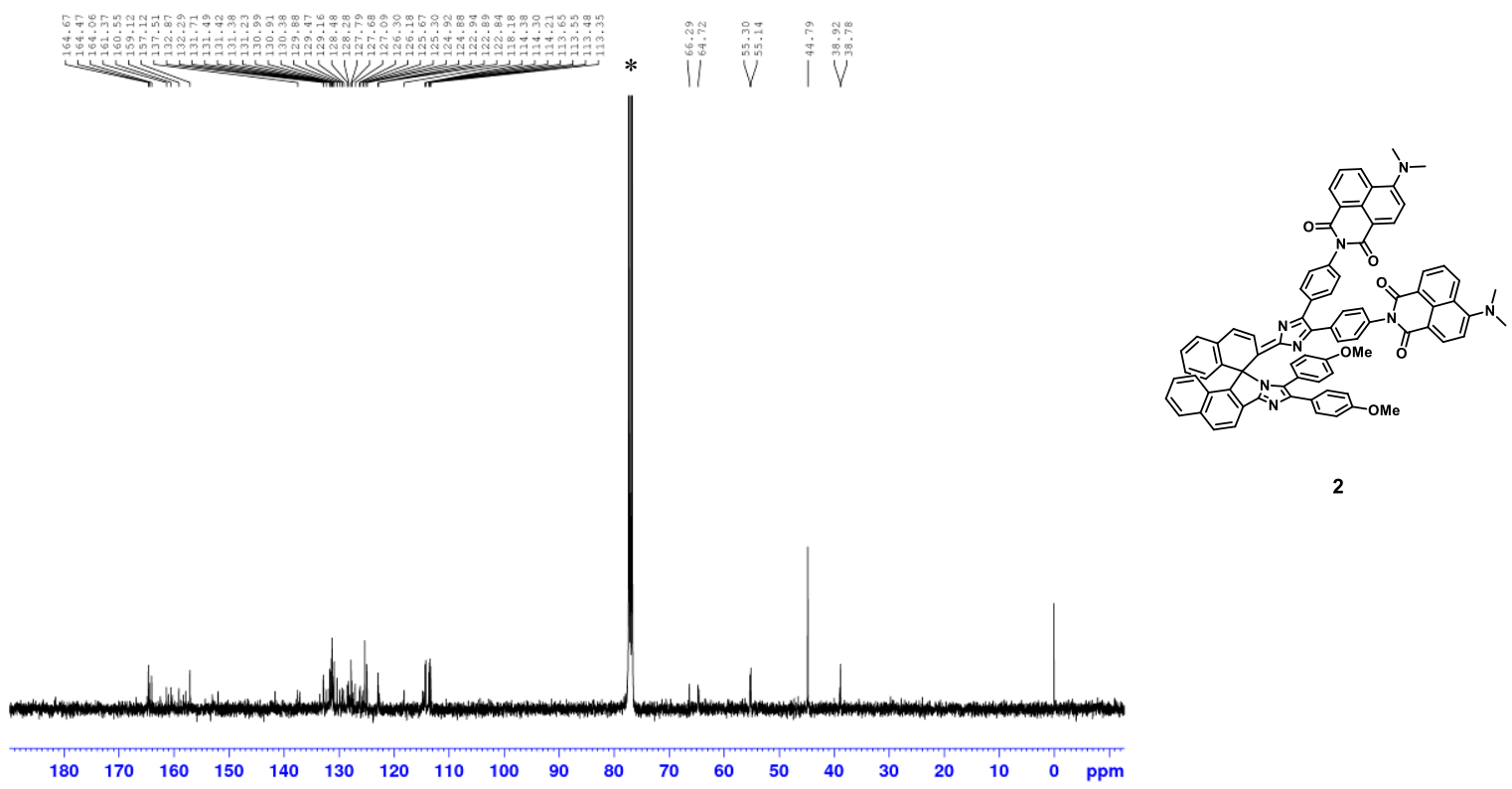

Figure S19. ${ }^{13} \mathrm{C}$ NMR spectrum of 2 in $\mathrm{CDCl}_{3}$ (* solvent peaks). 


\section{HR-ESI-TOF MS Spectra}

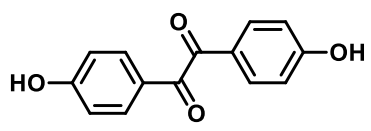

$1 \mathrm{a}$

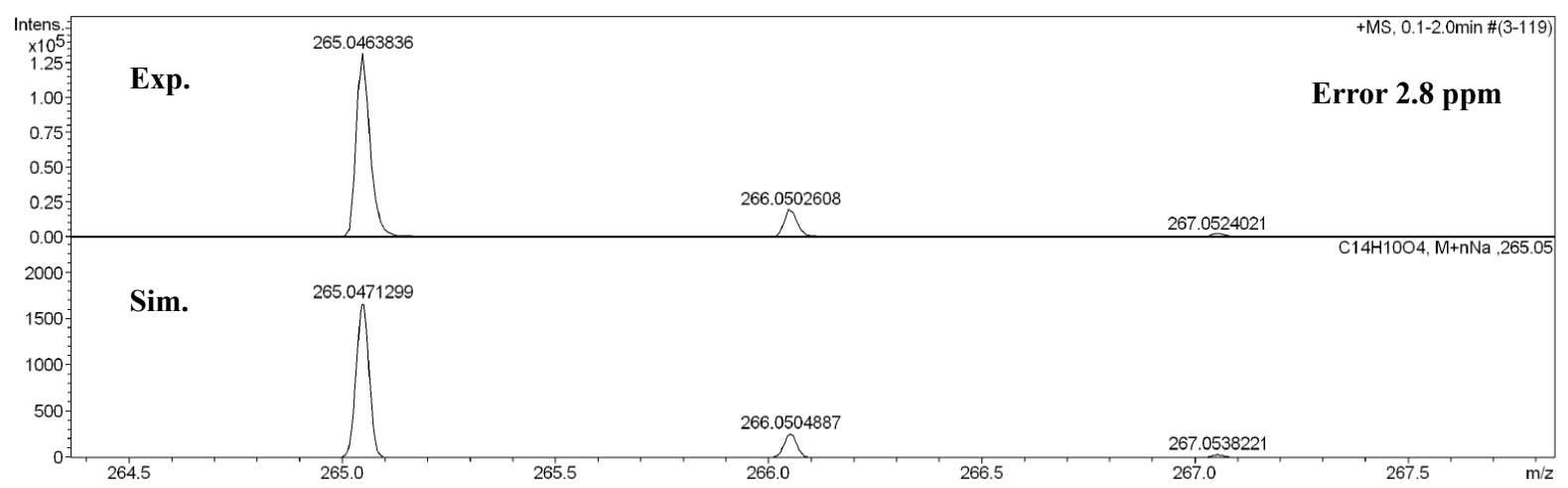

Figure S20. HR-ESI-TOF MS spectra of 4,4'-dihydroxy-benzil (1a).

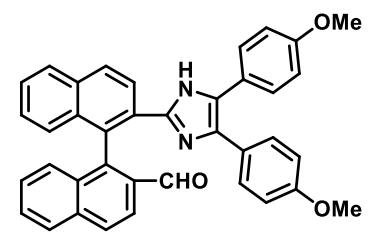

1c

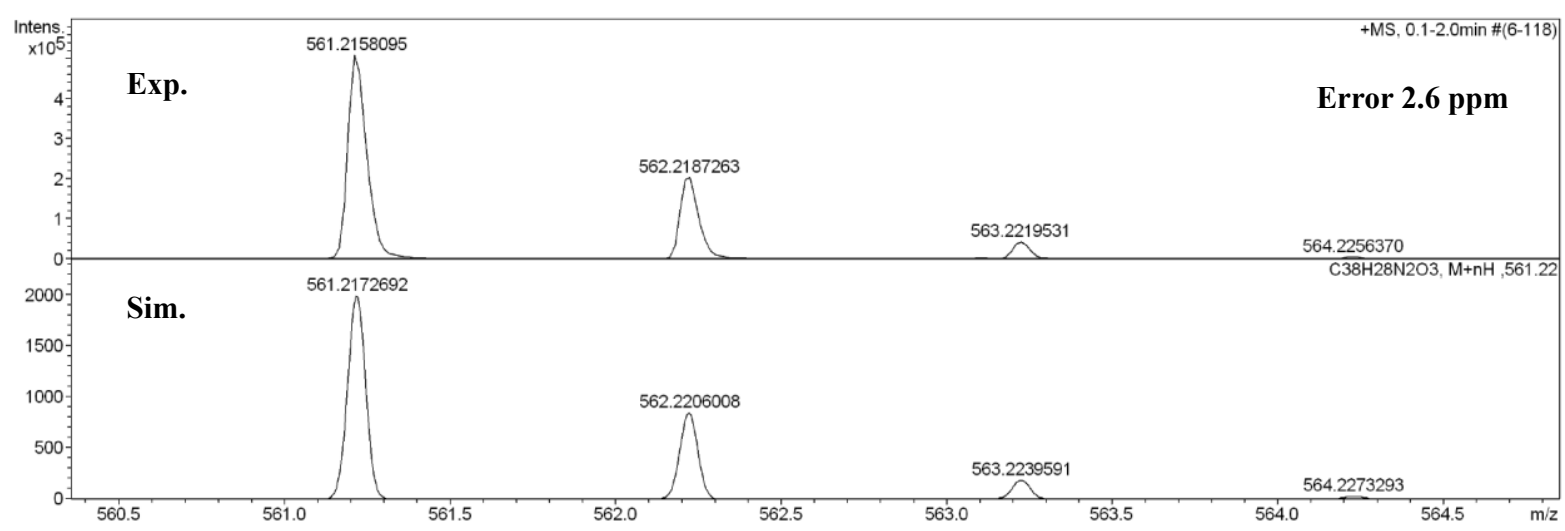

Figure S21. HR-ESI-TOF MS spectra of 2'-(4,5-bis(4-methoxyphenyl)-1H-imidazol-2-yl)-[1,1'-binaphthalene]-2carbaldehyde (1c). 


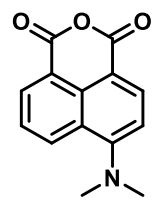

1d

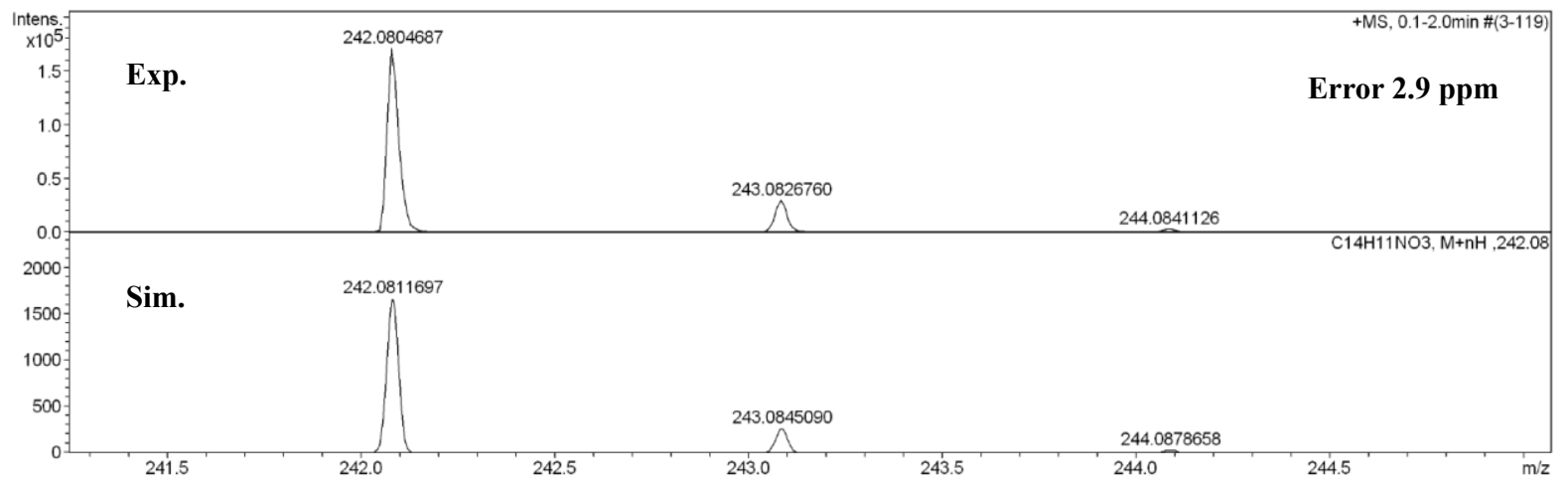

Figure S22. HR-ESI-TOF MS spectra of 4-(dimethylamino)-1,8-naphthalic anhydride (1d).

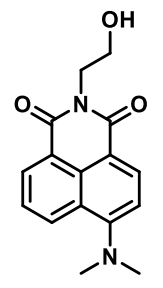

$1 e$

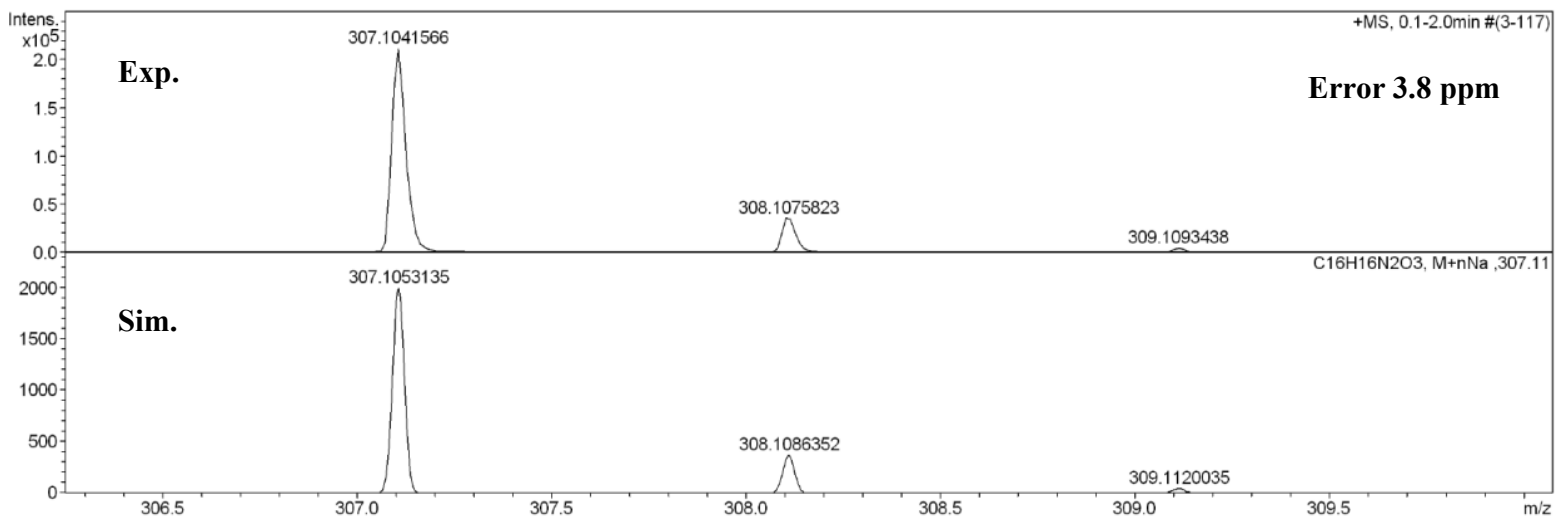

Figure S23. HR-ESI-TOF MS spectra of 6-(dimethylamino)-2-(2-hydroxyethyl)-1H-benzo[de]isoquinoline-1,3(2H)dione (1e). 


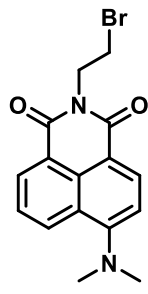

$1 f$

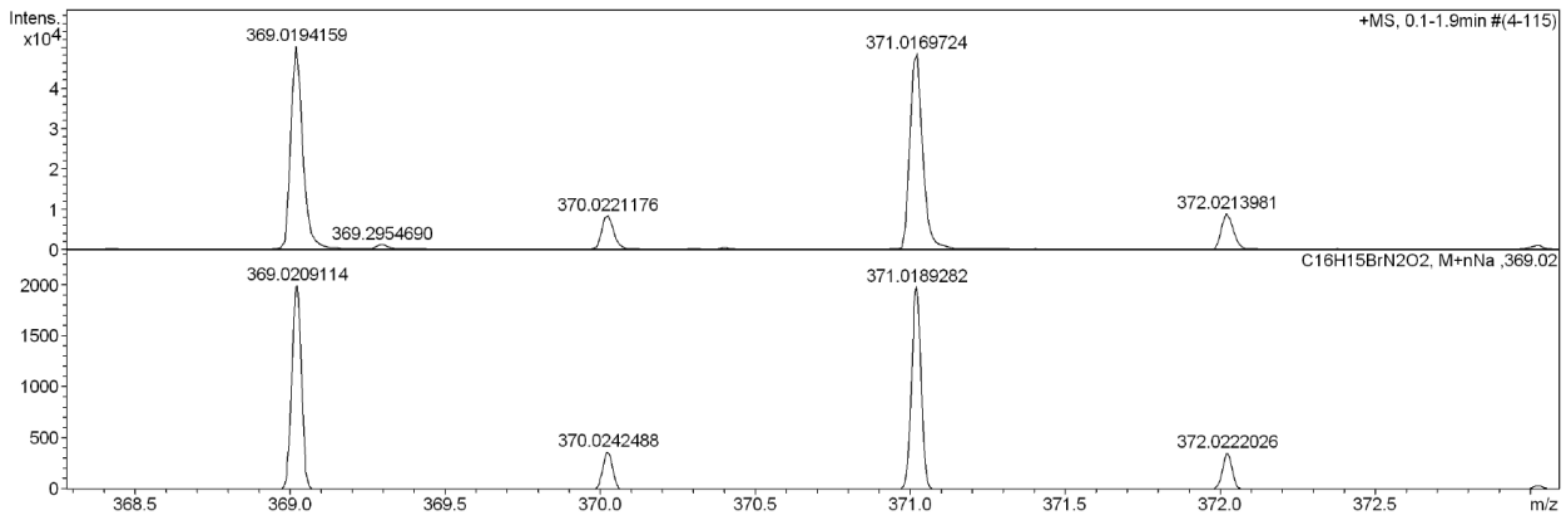

Figure S24. HR-ESI-TOF MS spectra of 2-(2-bromoethyl)-6-(dimethylamino)-1H-benzo[de]isoquinoline-1,3(2H)-dione (1f).
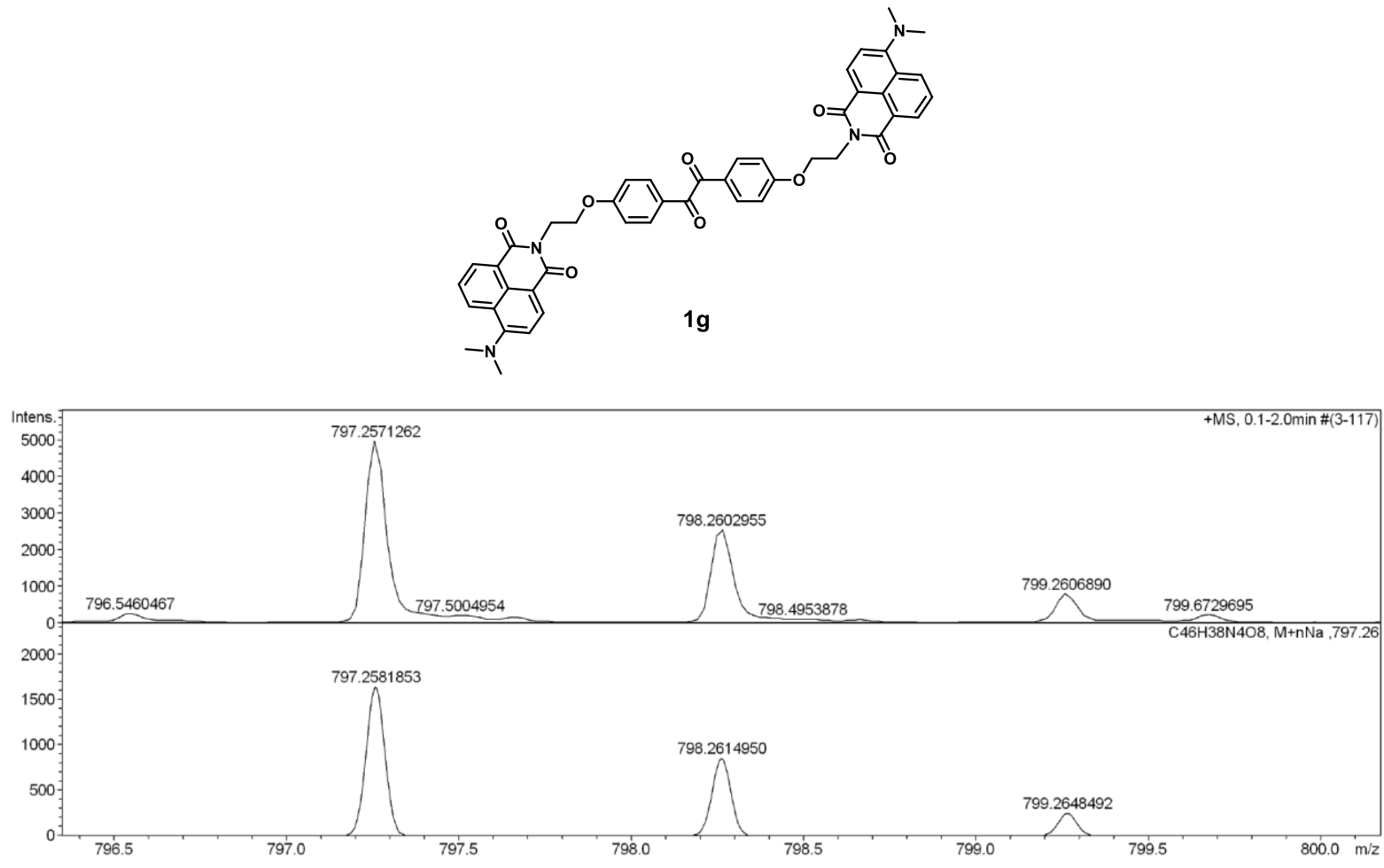

Figure S25. HR-ESI-TOF MS spectra of 2,2'-(((oxalylbis(4,1-phenylene))bis(oxy))bis(ethane-2,1-diyl))bis(6(dimethylamino)-1H-benzo[de]isoquinoline-1,3(2H)-dione) (1g). 

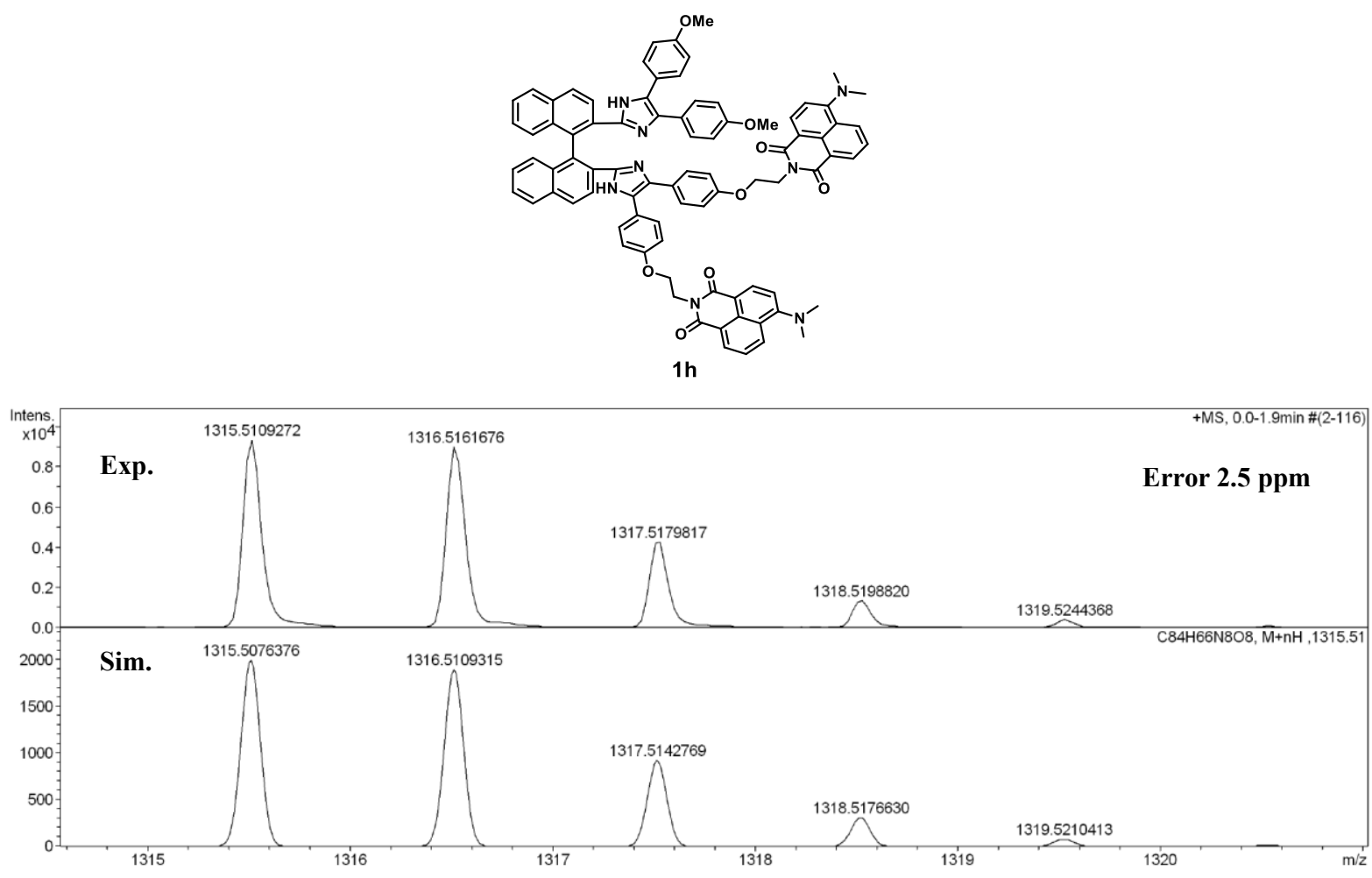

Figure S26. HR-ESI-TOF MS spectra of 2,2'-((((2-(2'-(4,5-bis(4-methoxyphenyl)-1H-imidazol-2-yl)-[1,1'-binaphthalen]2-yl)-1H-imidazole-4,5-diyl)bis(4,1-phenylene))bis(oxy))bis(ethane-2,1-diyl))bis(6-(dimethylamino)-1Hbenzo $[d e]$ isoquinoline-1,3(2H)-dione) (1h).

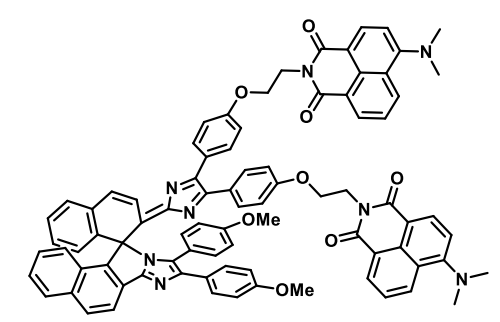

1

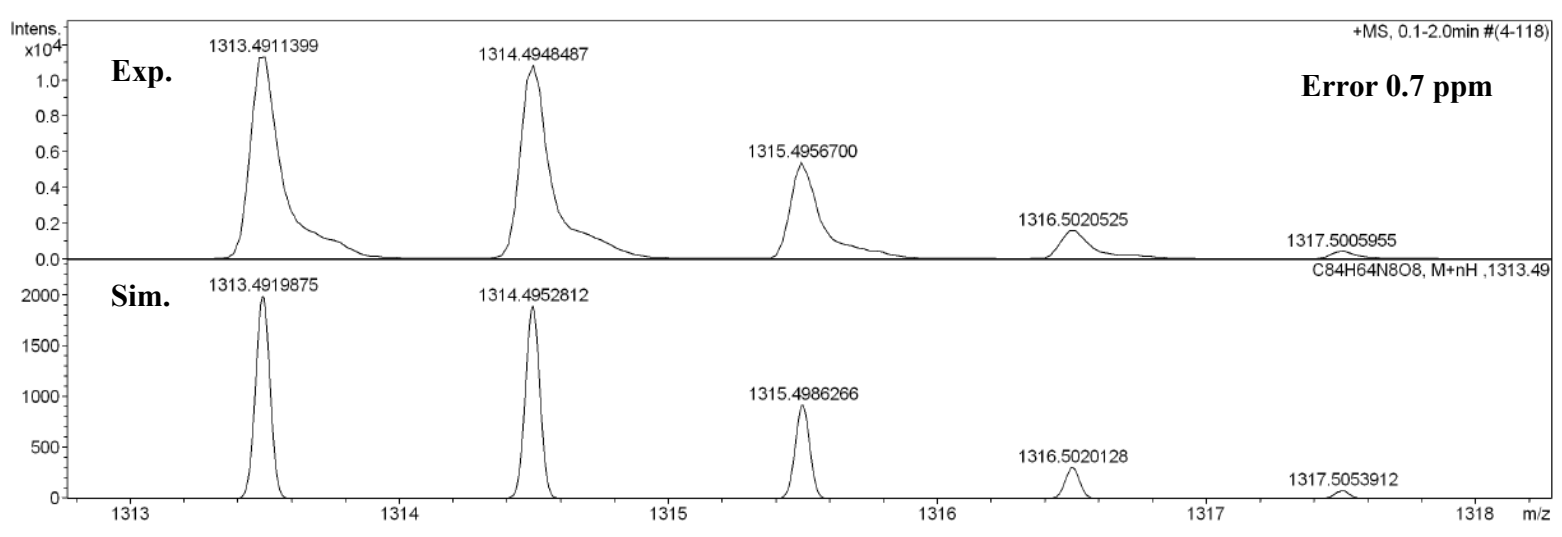

Figure S27. HR-ESI-TOF MS spectra of 1. 


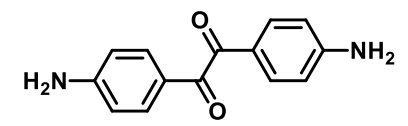

2b

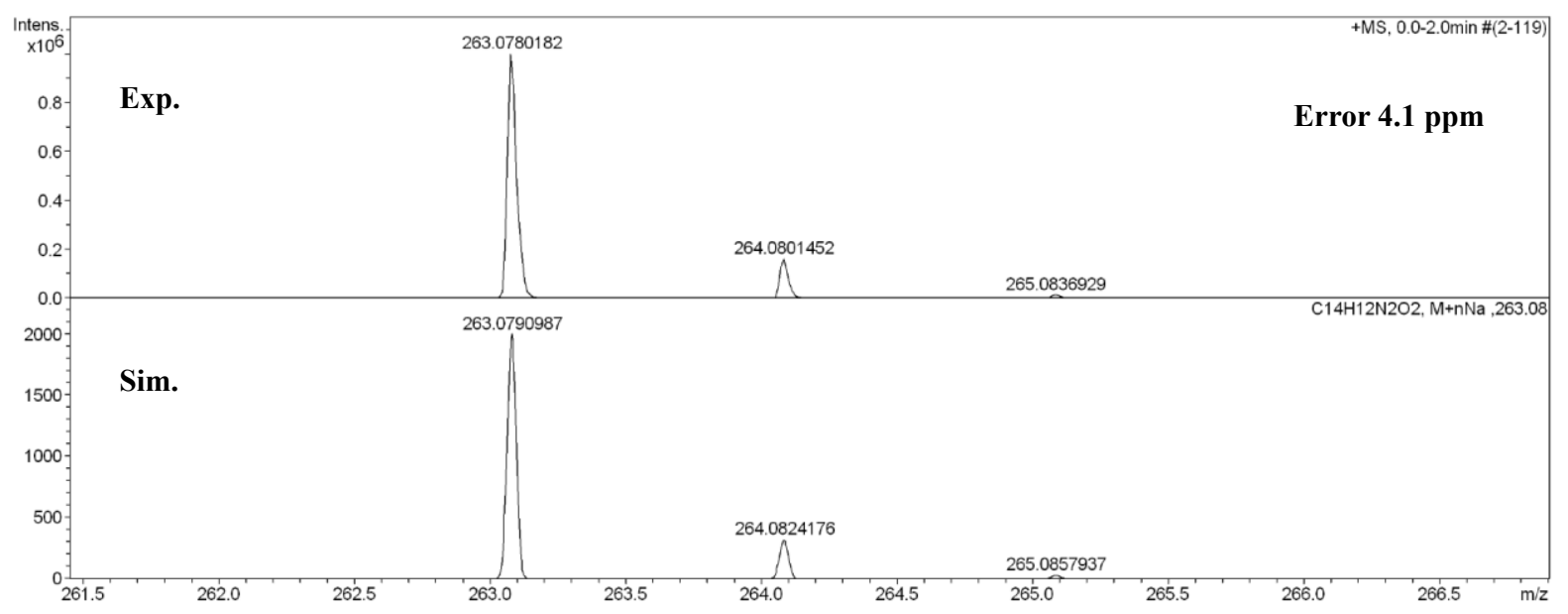

Figure S28. HR-ESI-TOF MS spectra of 4,4'-diamino-benzil (2b).

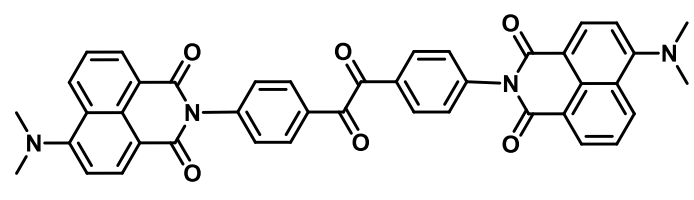

2c

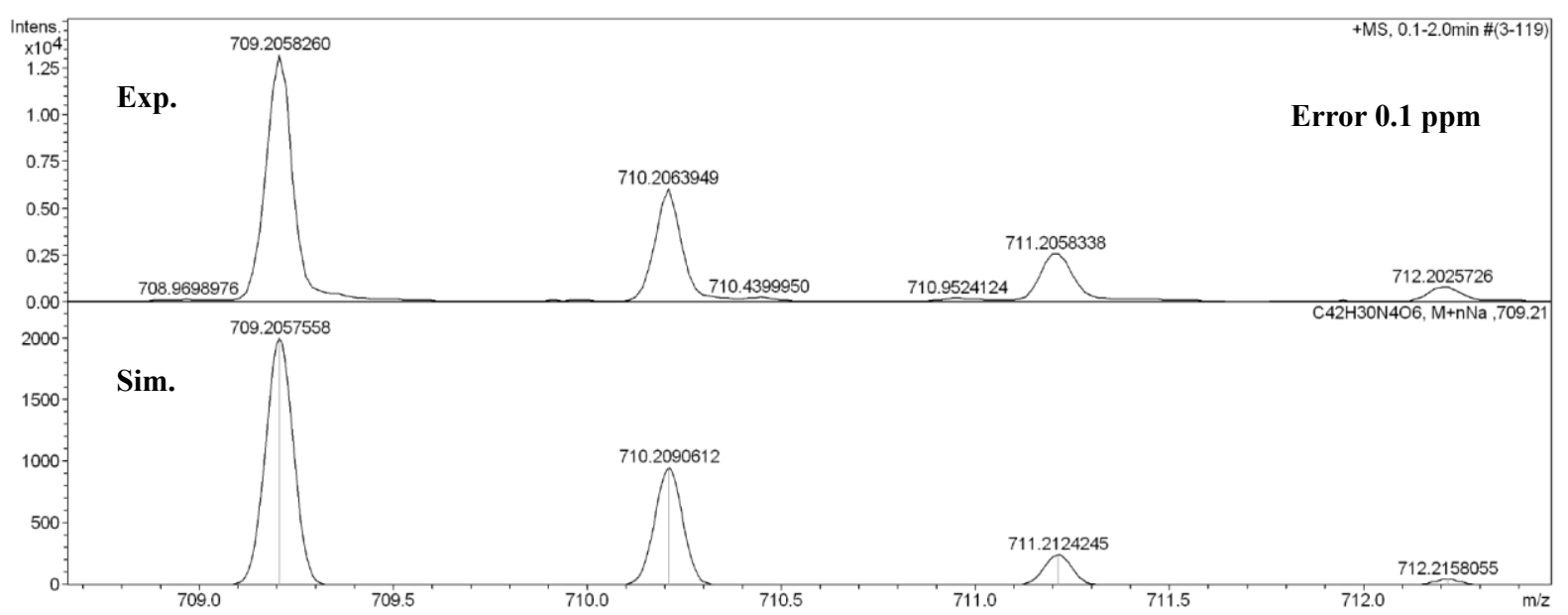

Figure S29. HR-ESI-TOF MS spectra of 2,2'-(oxalylbis(4,1-phenylene))bis(6-(dimethylamino)-1H-benzo[de] isoquinoline-1,3(2H)-dione) (2c). 


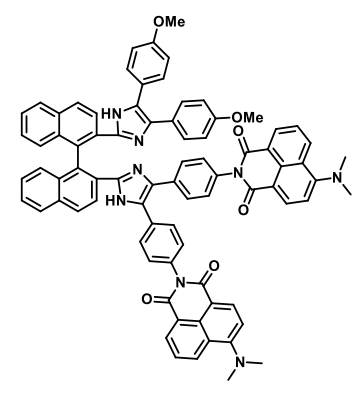

2d

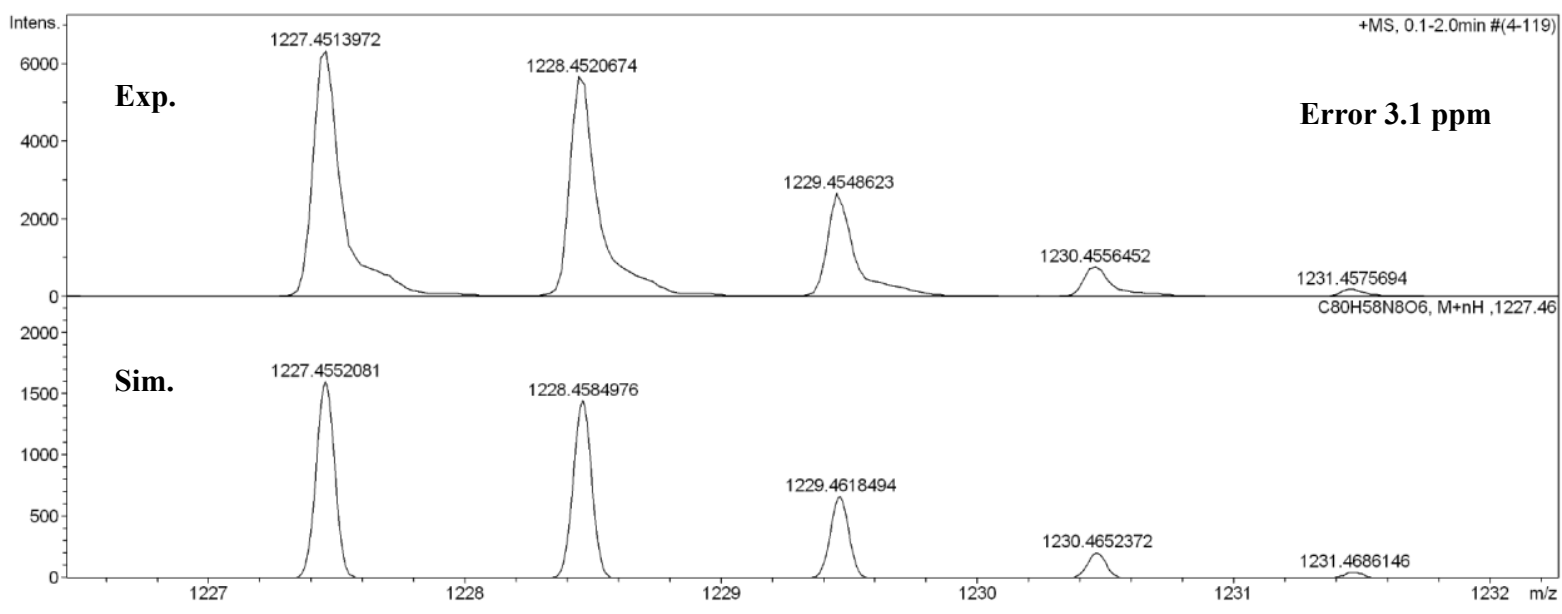

Figure S30. HR-ESI-TOF MS spectra of 2,2'-((2-(2'-(4,5-bis(4-methoxyphenyl)-1H-imidazol-2-yl)-[1,1'-binaphthalen]2-yl)-1H-imidazole-4,5-diyl)bis(4,1-phenylene))bis(6-(dimethylamino)-1H-benzo[de]isoquinoline-1,3(2H)-dione) (2d).
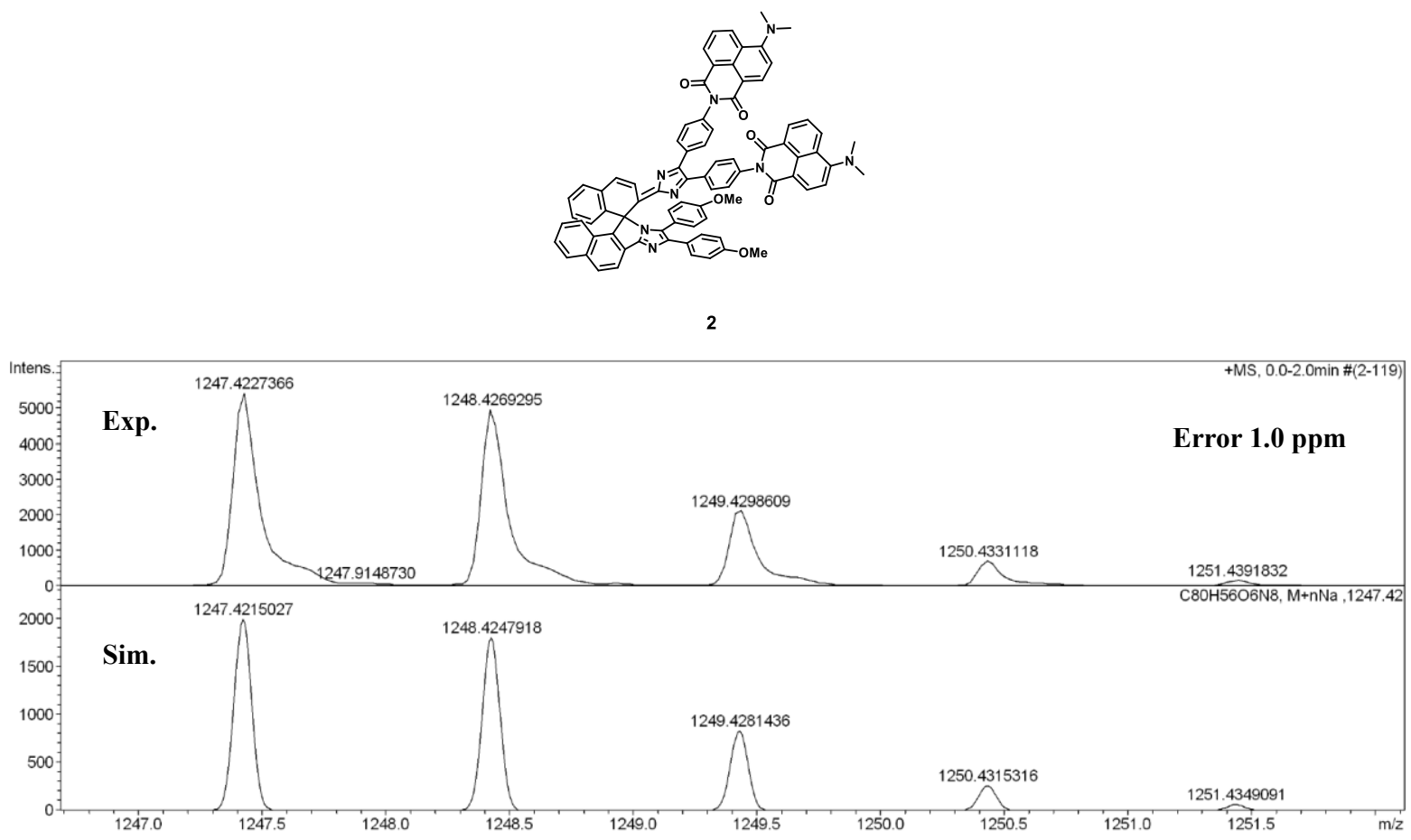

Figure S31. HR-ESI-TOF MS spectra of 2. 


\section{HPLC Chromatograms}
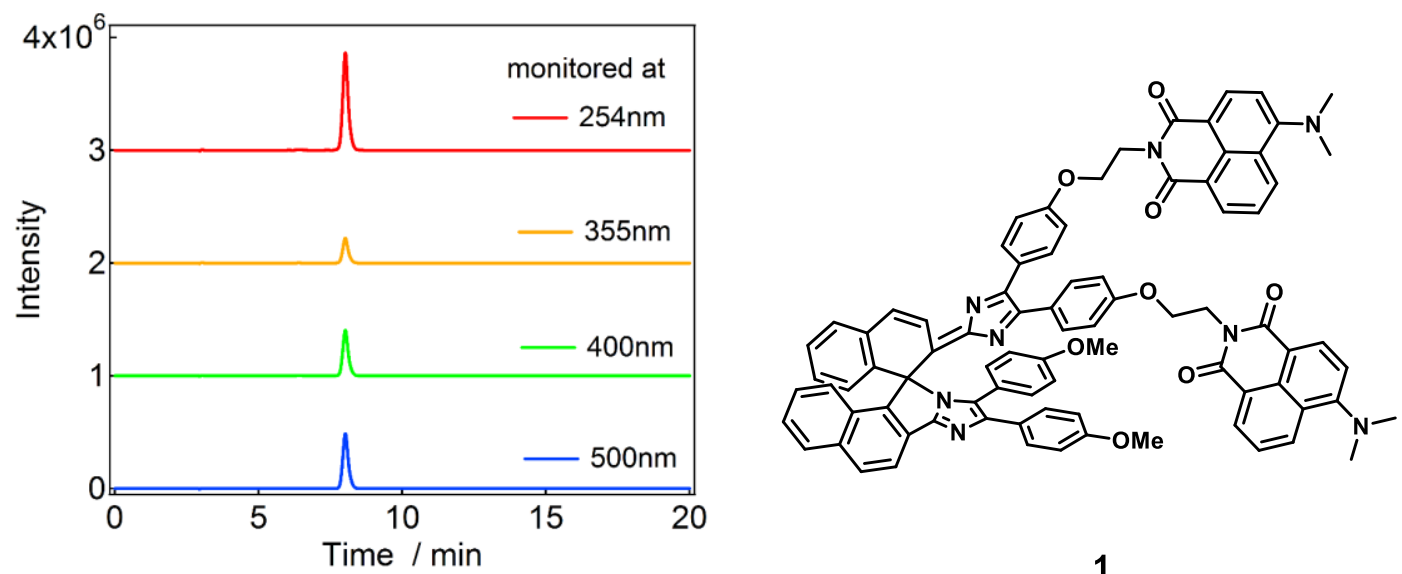

1

Figure S32. HPLC chromatograms of 1; $99 \%$ purity. HPLC analysis was performed using a reverse phase analytical column (Mightysil RP18, 25cm $\times 4.6 \mathrm{~mm}, 5 \mu \mathrm{m}$ particle) from Kanto Chemical Industries, equipped with a UV detector; the mobile phase was $\mathrm{CH}_{3} \mathrm{CN} / \mathrm{THF}=50 / 1$ with a flow rate of $1.0 \mathrm{~mL} / \mathrm{min}$ (detection wavelength; 254, 355, 400 and $500 \mathrm{~nm}$ ).
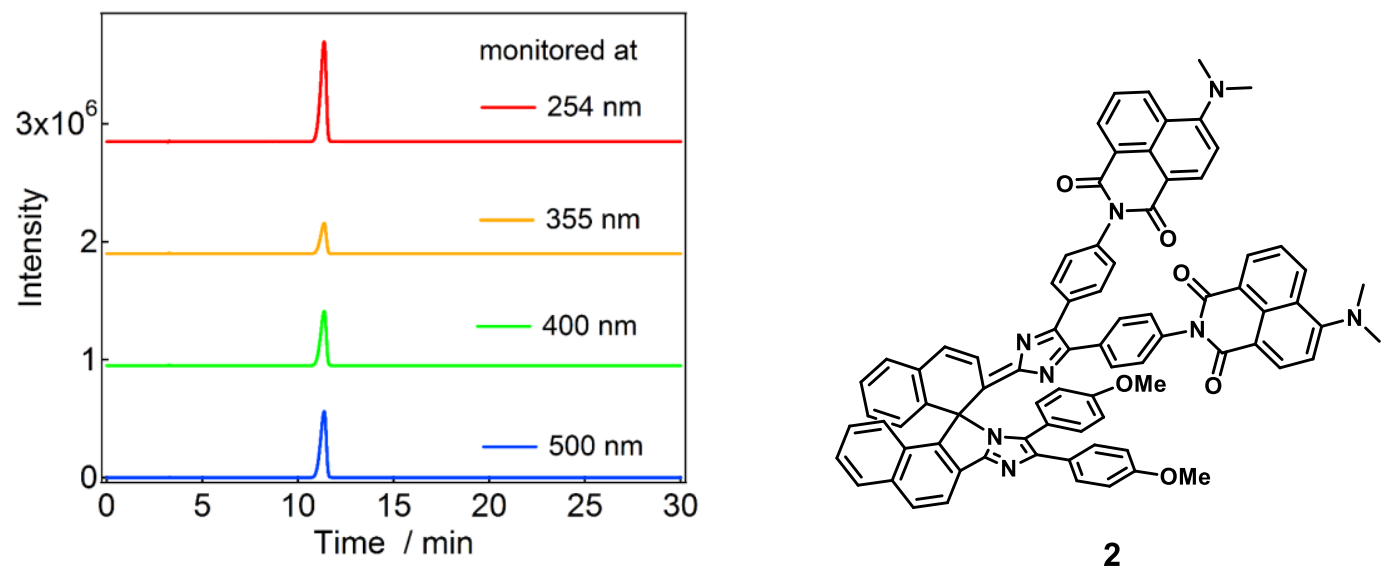

Figure S33. HPLC chromatograms of 2; $99 \%$ purity. HPLC analysis was performed using a reverse phase analytical column (Mightysil RP18, $25 \mathrm{~cm} \times 4.6 \mathrm{~mm}, 5 \mu \mathrm{m}$ particle) from Kanto Chemical Industries, equipped with a UV detector; the mobile phase was $\mathrm{CH}_{3} \mathrm{CN} / \mathrm{H}_{2} \mathrm{O}=10 / 1$ with a flow rate of $1.0 \mathrm{~mL} / \mathrm{min}$ (detection wavelength; 254, 355, 400 and $500 \mathrm{~nm}$ ). 


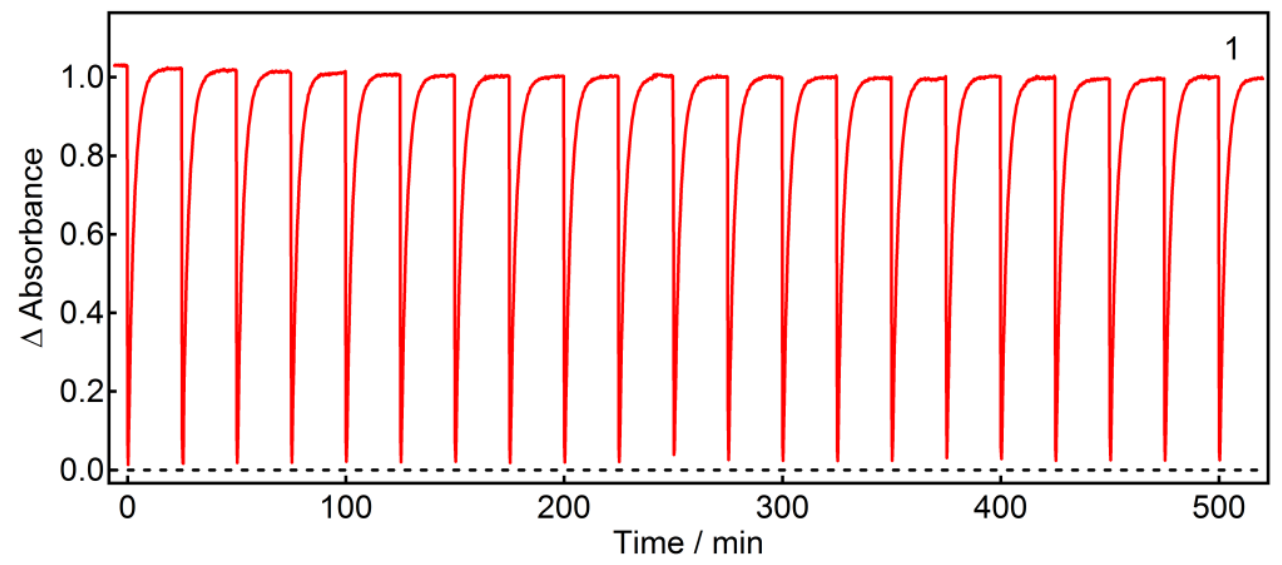

Figure S34. Photoisomerization cycles of 1 upon 525-nm light ( $83 \mathrm{~mW}$ ) irradiation in Ar-bubbled benzene $\left(3.0 \times 10^{-5} \mathrm{M}\right)$ at $25^{\circ} \mathrm{C}$.

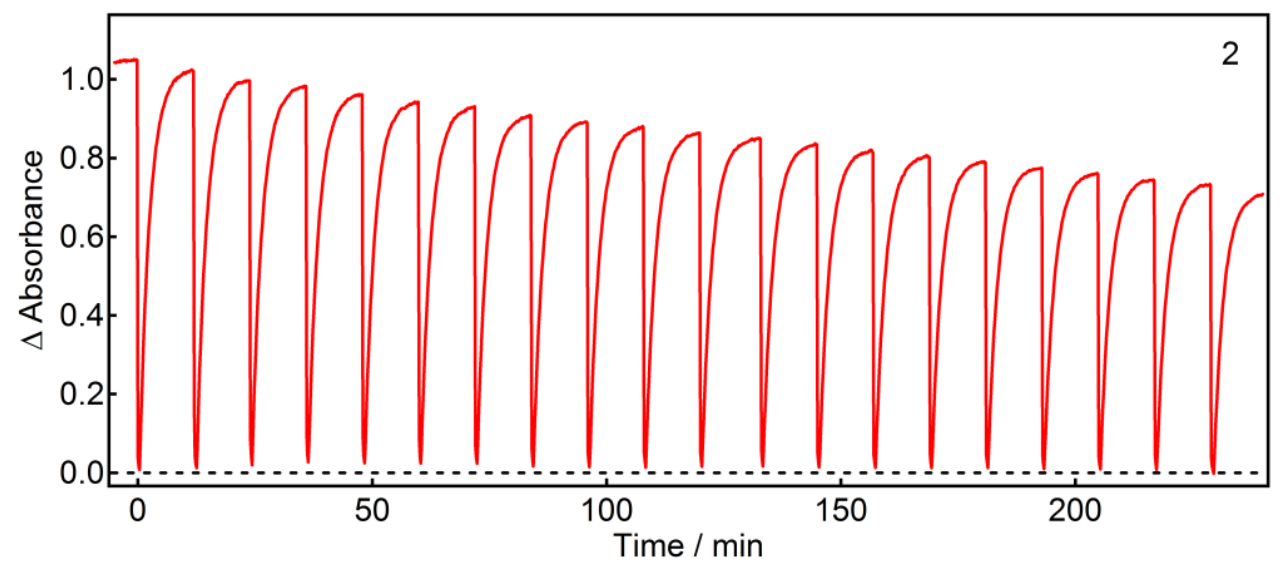

Figure S35. Photoisomerization cycles of 2 upon 525-nm light ( $83 \mathrm{~mW}$ ) irradiation in Ar-bubbled benzene $\left(3.0 \times 10^{-5} \mathrm{M}\right)$ at $25^{\circ} \mathrm{C}$. 


\section{Estimation of Conversion Efficiency}

The conversion efficiency from the colored to the colorless forms of $\mathbf{1}$ and $\mathbf{2}$ were estimated by using 1,2-bis(2methylbenzo[b]thiophene-3-yl)-perfluorocyclopentene (DAE) as a standard. ${ }^{\mathrm{S2}} \mathrm{We}$ prepared the solutions of $\mathbf{1}, \mathbf{2}$ and DAE matched the absorbance at $517 \mathrm{~nm}$ in benzene. The changes in the absorbance upon 517-nm laser irradiation $\left(\Delta \mathrm{OD}_{1}, \Delta \mathrm{OD}_{2}\right.$ and $\left.\Delta \mathrm{OD}_{\mathrm{DAE}}\right)$ were plotted against the laser power to estimate the $\Delta \mathrm{OD}$ values per unit laser power. The conversion efficiency $\left(\varphi_{1}\right.$ and $\left.\varphi_{2}\right)$ were estimated to be 0.07 and 0.19 , respectively, from the following equations,

$$
\begin{gathered}
\frac{\varphi_{1}}{\varphi_{D A E}}=\frac{\Delta O D_{1}}{\varepsilon_{1}} \times \frac{\varepsilon_{D A E}}{\Delta O D_{D A E}} \\
\frac{\varphi_{2}}{\varphi_{D A E}}=\frac{\Delta O D_{2}}{\varepsilon_{2}} \times \frac{\varepsilon_{D A E}}{\Delta O D_{D A E}}
\end{gathered}
$$

where $\varphi_{\mathrm{DAE}}(0.35)$ is the quantum yield of the open-ring reaction of DAE, $\varepsilon 1\left(2.27 \times 10^{4} \mathrm{M}^{-1} \mathrm{~cm}^{-1}\right), \varepsilon 1\left(2.51 \times 10^{4} \mathrm{M}^{-1} \mathrm{~cm}^{-1}\right)$ and $\varepsilon \operatorname{DAE}\left(0.91 \times 10^{4} \mathrm{M}^{-1} \mathrm{~cm}^{-1}\right)$ are absorption coefficients at $517 \mathrm{~nm}$ for 1-O, 2-O and the closed-ring form of DAE, respectively.

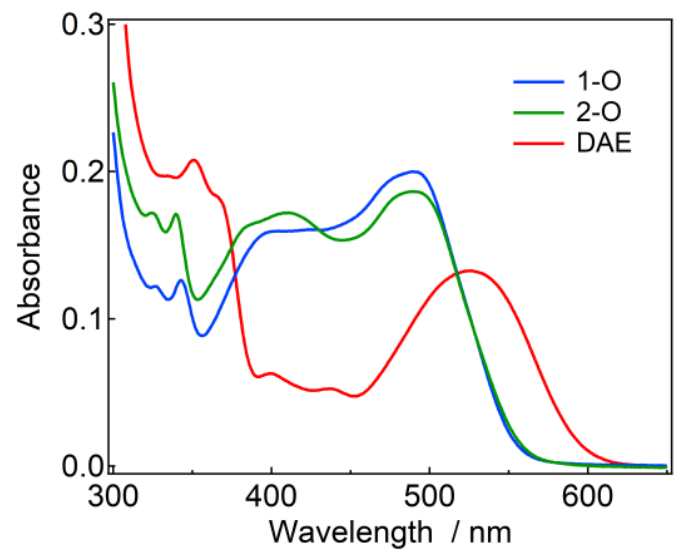

Figure S36. Absorption spectra of 1-O, 2-O and DAE in benzene. 

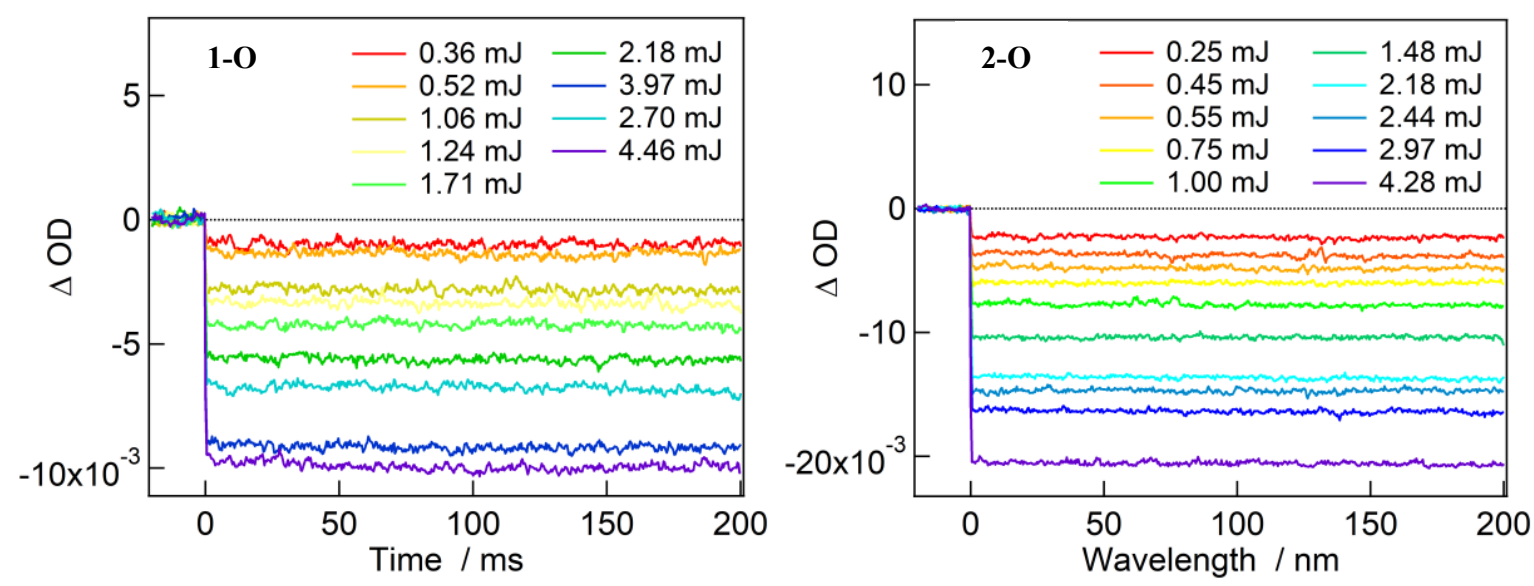

Figure S37. Laser intensity dependence of the time profiles of transient absorbance of 1-O and 2-O in benzene at $298 \mathrm{~K}$ monitored at $517 \mathrm{~nm}$. (excitation wavelength: $517 \mathrm{~nm}$, pulse width: $5 \mathrm{~ns}$ )

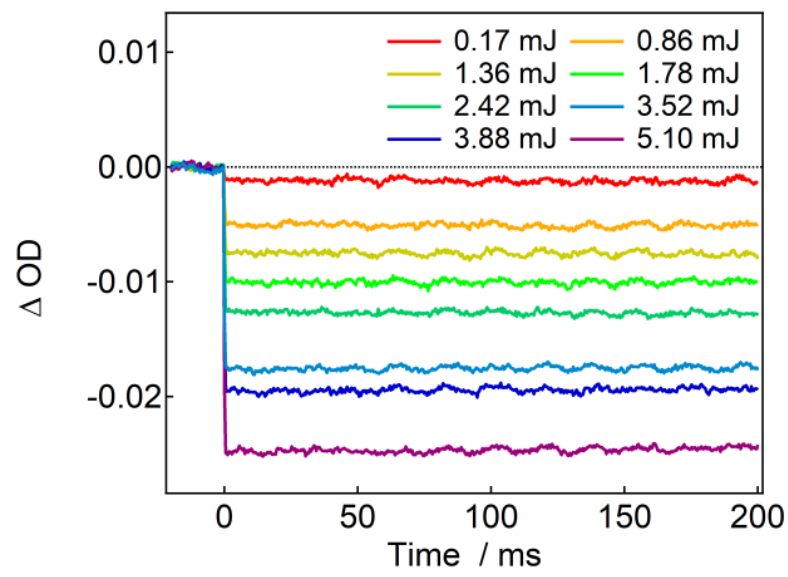

Figure S38. Laser intensity dependence of the time profiles of transient absorbance of the closed-ring isomer of DAE in benzene at $298 \mathrm{~K}$ monitored at $517 \mathrm{~nm}$. (excitation wavelength: $517 \mathrm{~nm}$, pulse width: $5 \mathrm{~ns}$ ) 


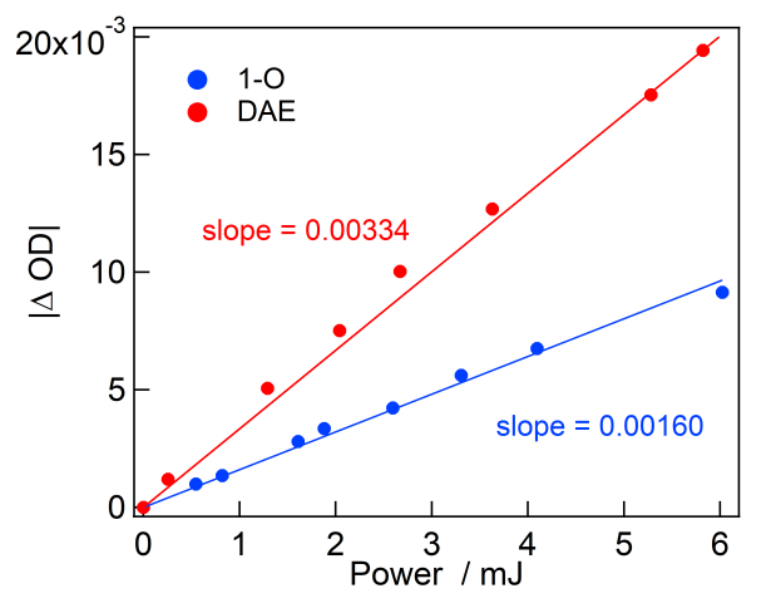

Figure S39. Linear relationship between the laser power and the $\triangle \mathrm{OD}$ of 1-O and DAE monitored at $517 \mathrm{~nm}$.

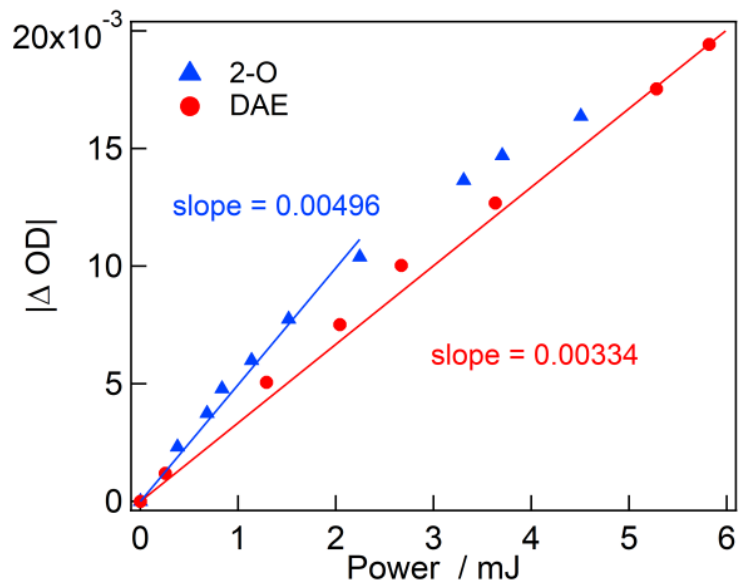

Figure S40. Linear relationship between the laser power and the $\triangle \mathrm{OD}$ of 2-O and DAE monitored at $517 \mathrm{~nm}$. 


\section{Fluorescence Lifetime Measurement}

\subsection{Fluorescence Lifetime Measurement of $\mathbf{1}$ and $\mathbf{2}$}

(a)

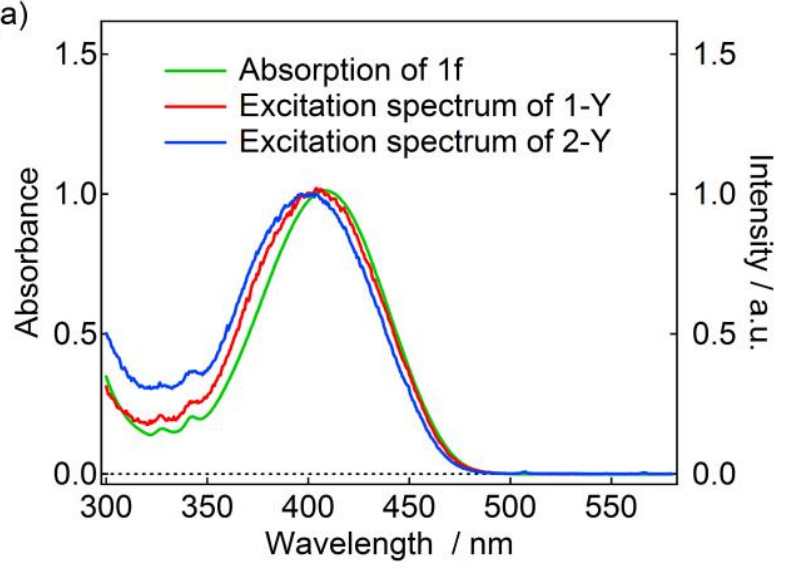

(b)

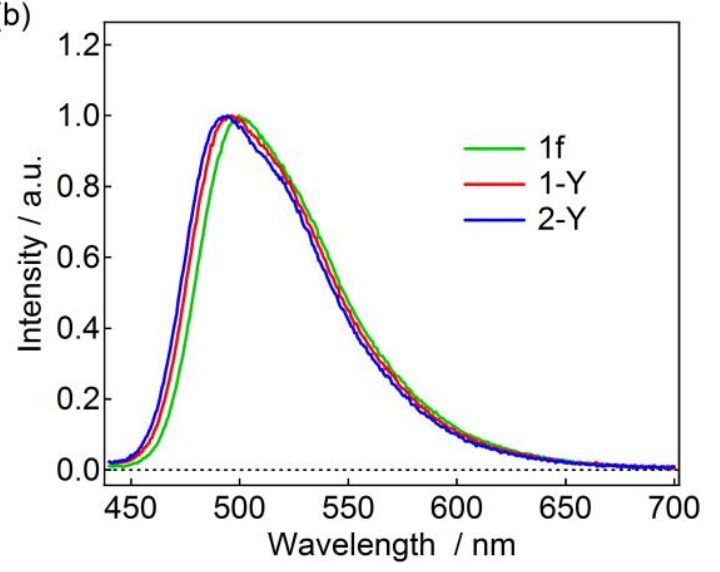

Figure S41. (a) Absorption spectrum of 1f and Excitation spectra of 1-Y and 2-Y, (b) Fluorescence spectra of 1f, 1-Y and 2-Y in benzene.

(a)
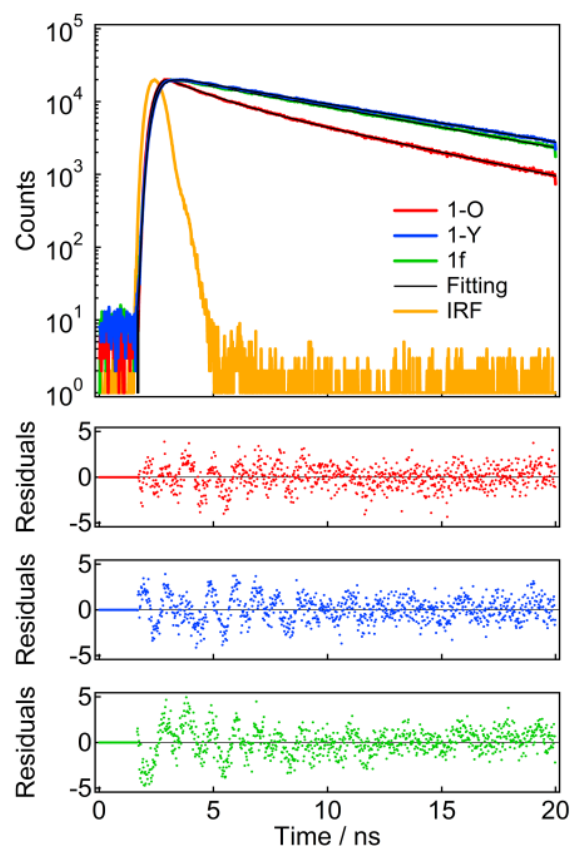

(b)
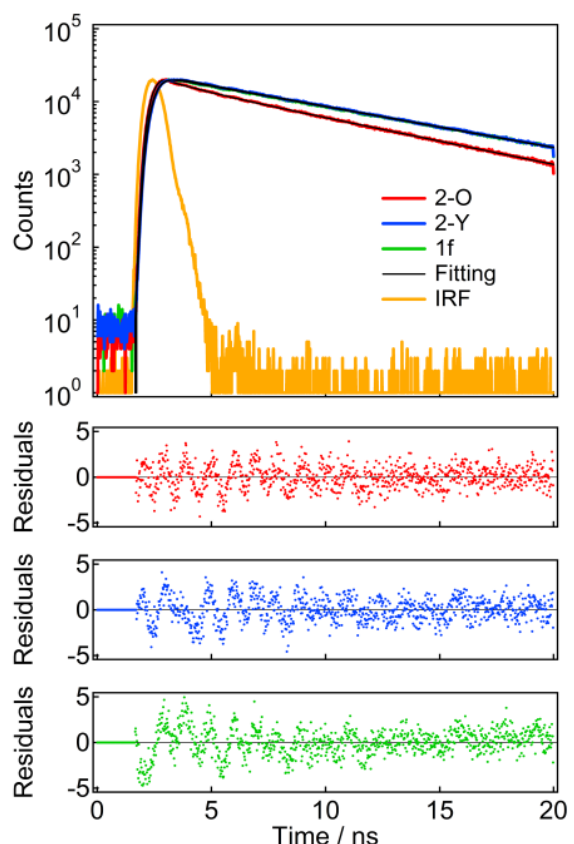

Figure S42. Fluorescence lifetimes of (a) 1 and (b) $2\left(\lambda_{\text {ex. }}=405 \mathrm{~nm}\right.$ and $\left.\lambda_{\text {obs. }}=500 \mathrm{~nm}\right)$ in benzene. The lifetimes of the orange forms and the yellow forms were measured at $293 \mathrm{~K}$ and at $250 \mathrm{~K}$, respectively. 
Table S1. Fluorescence Decay Time Constants (contribution in \%) of 1 and 2

\begin{tabular}{cccc}
\hline & $\tau_{1}(\mathrm{~ns})$ & $\tau_{2}(\mathrm{~ns})$ & $\tau_{3}(\mathrm{~ns})$ \\
\hline \multirow{2}{*}{$1-\mathrm{O}$} & 0.12 & 2.11 & 6.79 \\
& $(57.2 \%)$ & $(19.6 \%)$ & $(23.2 \%)$ \\
\multirow{2}{*}{$1-\mathrm{Y}$} & - & 0.40 & 8.24 \\
& & $(26.6 \%)$ & $(73.4 \%)$ \\
$2-\mathrm{O}$ & 0.08 & 1.51 & 6.74 \\
& $(69.2 \%)$ & $(5.3 \%)$ & $(25.5 \%)$ \\
$2-\mathrm{Y}$ & - & 0.47 & 7.63 \\
& & $(24.8 \%)$ & $(75.2 \%)$ \\
1f & - & - & 7.70 \\
\hline
\end{tabular}

\subsection{Fluorescence Lifetime Measurement of the mixture solution of $\mathbf{1 f}$ and BN-ImD}

we checked the FRET efficiency of the mixture solution of BN-ImD and the naphthalimide unit (1f). The solutions of $\mathbf{1 f}(2.3 \times$ $\left.10^{-4} \mathrm{M}\right)$ and the mixture of $1 \mathrm{f}\left(2.3 \times 10^{-4} \mathrm{M}\right)$ and $\mathrm{BN}-\mathrm{ImD}\left(2.3 \times 10^{-4} \mathrm{M}\right.$ or $\left.2.3 \times 10^{-3} \mathrm{M}\right)$ were prepared for the fluorescence lifetime measurement. Because the mixture solution is highly concentrated, and $1 \mathrm{~mm}$ optical cell was used for the measurement. The fluorescence lifetime of the mixture consists of the two decay components, and the short one decreases to ca. $1 \mathrm{~ns}$ with increasing the concentration of BN-ImD. This decrement of the lifetime indicates the fluorescence of $\mathbf{1 f}$ is also quenched by FRET in the mixture solution, but the FRET efficiency $\left(1-\tau / \tau_{0}\right)$ of the mixture solution (estimated to be $\left.87 \%\right)$ is lower than those of 1-O and 2-O (ca. 99\%).

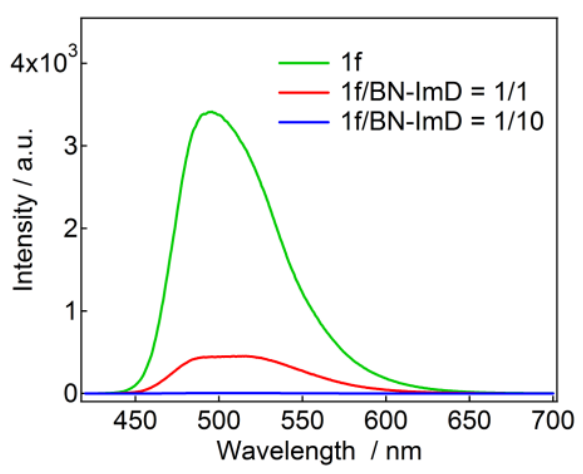

Figure S43. Fluorescence spectra of $\mathbf{1 f}$ and the mixture of $\mathbf{1 f}$ and $\mathrm{BN}-\operatorname{ImD}(1 \mathrm{f} / \mathrm{BN}-\operatorname{ImD}=1 / 1$ and $1 / 10)$ in benzene. 

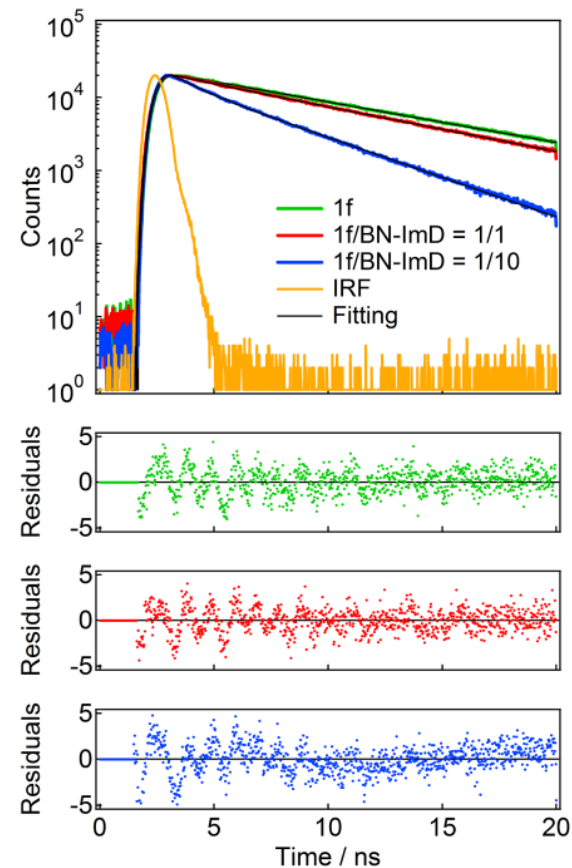

Figure S44. Fluorescence lifetimes of $\mathbf{1 f}$ and the mixture of $\mathbf{1 f}$ and $\mathrm{BN}-\mathrm{ImD}(1 \mathrm{f} / \mathrm{BN}-\mathrm{ImD}=1 / 1$ and $1 / 10)$ in benzene $\left(\lambda_{\mathrm{ex} .}=\right.$ $405 \mathrm{~nm}$ and $\lambda_{\text {obs. }}=500 \mathrm{~nm}$ ).

Table S2. Fluorescence Decay Time Constants (Contribution in \%) of $\mathbf{1 f}$ and the Mixture of $\mathbf{1 f}$ and BN-ImD in Benzene

\begin{tabular}{ccc}
\hline & $\tau_{1}(\mathrm{~ns})$ & $\mathrm{T}_{2}(\mathrm{~ns})$ \\
\hline \multirow{2}{*}{ 1f } & - & 7.80 \\
& 1.66 & 7.08 \\
$1 \mathrm{f} / \mathrm{BN}-\mathrm{ImD}=1 / 1$ & $(8.3 \%)$ & $(91.7 \%)$ \\
& 1.05 & 3.96 \\
$1 \mathrm{f} / \mathrm{BN}-\mathrm{ImD}=1 / 10$ & $(32.6 \%)$ & $(67.4 \%)$ \\
\hline
\end{tabular}




\section{Cyclic Voltammetry and Rehm-Weller Equation}

The energy gaps $\left(\Delta G^{0}\right)$ for the charge separation reaction between the $\mathrm{BN}-\mathrm{ImD}$ and the naphthalimide units were estimated by the following Rehm-Weller equation ${ }^{\mathrm{S} 3}$

$$
\Delta G^{o}=e\left(E_{D^{+} / D}-E_{A / A^{-}}\right)-\Delta E_{00}-\frac{e^{2}}{4 \pi \varepsilon_{0} \varepsilon R}
$$

where $e$ is the electron charge $\left(1.602 \times 10^{-19} \mathrm{C}\right), \varepsilon$ is the relative permittivity of the solvent (benzene $\left.=2.27\right), E_{D^{+} / D}$ is the first oxidation potential of the donor (1f), $E_{A / A^{-}}$is the first reduction potential of the accepter $(\mathrm{BN}-\mathrm{ImD}), \Delta E_{00}$ is the spectroscopic excited energy of the donor and $R$ is the distance between the donor and acceptor (herein, the distance between the center of the naphthalimide unit and the center of BN-ImD, estimated from the optimized structures by the semiempirical quantum chemical PM6 method for 1-O and 2-O). The oxidation and the reduction potentials were estimated from the results of the cyclic voltammetry of $\mathbf{1 f}$ and the colored and the colorless forms of BN-ImD. Because the oxidation and the reduction processes are irreversible, we roughly estimated the oxidation and the reduction potentials from the peak values (Figure S45). The results were summarized in Table S3 and suggest that the photoinduced electron transfer from the naphthalimide unit to the orange form of BN-ImD is energetically favorable $\left(\Delta G^{0}=-70 \mathrm{~kJ} / \mathrm{mol}\right)$.

Table S3. Spectroscopic Excited-State energy of $1 \mathbf{f}\left(\Delta E_{00}\right)$, the Oxidation Potential of $\mathbf{1 f}\left(E_{D^{+} / D}\right)$, the Reduction Potential of $\mathrm{BN}-\mathrm{ImD}\left(E_{A / A^{-}}\right)$, the Distance Between the Center of the Naphthalimide Unit and the Center of the BN-ImD Unit $(R)$ and the estimated Gibbs Free Energies $\left(\Delta G^{0}\right)$.

\begin{tabular}{cccccc}
\hline & $\begin{array}{c}\Delta E_{00} \\
{[\mathrm{eV}]}\end{array}$ & $\begin{array}{c}E_{D^{+} / D} \\
{[\mathrm{~V}]}\end{array}$ & $\begin{array}{c}E_{A / A^{-}} \\
{[\mathrm{V}]}\end{array}$ & $\begin{array}{c}R \\
{[\AA]}\end{array}$ & $\begin{array}{c}\Delta G^{0} \\
{[\mathrm{~kJ} / \mathrm{mol}]}\end{array}$ \\
\hline $\mathbf{1 - O}$ & 2.69 & 0.70 & -1.27 & 12.7 & -70 \\
$\mathbf{1 - Y}$ & 2.69 & 0.70 & -1.97 & 12.8 & -2.5 \\
$\mathbf{2 - O}$ & 2.69 & 0.70 & -1.27 & 10.1 & -70 \\
$\mathbf{2 - Y}$ & 2.69 & 0.70 & -1.97 & 10.1 & -2.6 \\
\hline
\end{tabular}

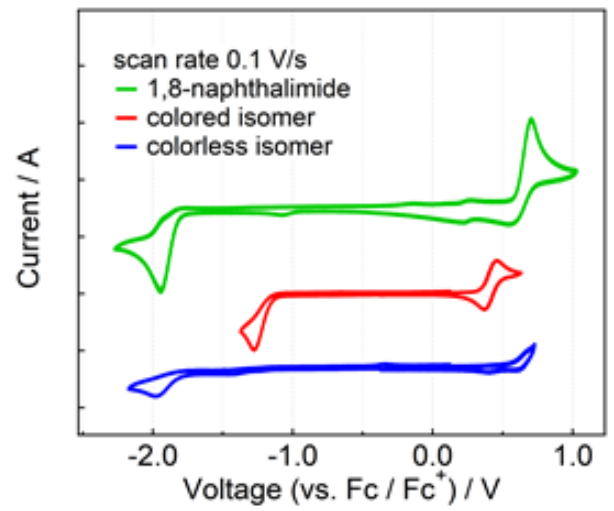

Figure S45. Cyclic voltammograms of $\mathbf{1 f}(1.15 \mathrm{mM})$ and the colored and the colorless isomers of BN-ImD $(0.96 \mathrm{mM})$ in dichloromethane containing $0.1 \mathrm{M} \mathrm{TBAPF}_{6}$ as the supporting electrolyte (potential scan rate $=100 \mathrm{mV} / \mathrm{s}$ ). 


\section{Kinetics of Thermal Back Reaction}

Continuous vis light $\left(400-700 \mathrm{~nm}, 260 \mathrm{~mW} / \mathrm{cm}^{2}\right)$ was irradiated to the solution of the compounds in a quartz optical cuvette (optical path length $=10 \mathrm{~mm}$ ) using a xenon lamp (LAX-100, Asahi Spectra Co., Ltd.). The transient absorption spectra and the time variation were recorded on a USB 4000 multichannel detector (Ocean Optics, Inc.). The power of the excitation light was measured using a Nova laser power meter equipped with a power thermal sensor 30A-P-17 (Ophir Optronics Solutions Ltd.). A CUV-qpod (Ocean Optics, Inc.) equipped with a TC 125 temperature controller (Quantum Northwest Inc.) was used as a cuvette holder. The probe beam from a deuterium and a halogen lamps, a DH-2000-BAL (Ocean Optics, Inc.), was guided with a QP-600-1-SR optical fiber (Ocean Optics, Inc.).

Table S4. First Order Rate Constants for the Thermal Back Reaction of $\mathbf{1}$ and $\mathbf{2}$ in Benzene

\begin{tabular}{ccc}
\hline $\mathrm{T} /{ }^{\circ} \mathrm{C}$ & $k / \mathrm{s}^{-1}(\mathbf{1})$ & $k / \mathrm{s}^{-1}(\mathbf{2})$ \\
\hline 15 & $1.357 \times 10^{-3}$ & $4.200 \times 10^{-3}$ \\
20 & $3.244 \times 10^{-3}$ & $7.939 \times 10^{-3}$ \\
25 & $6.313 \times 10^{-3}$ & $1.460 \times 10^{-2}$ \\
30 & $1.155 \times 10^{-2}$ & $2.660 \times 10^{-2}$ \\
35 & $2.136 \times 10^{-2}$ & $4.642 \times 10^{-2}$ \\
40 & $3.744 \times 10^{-2}$ & $8.059 \times 10^{-2}$ \\
45 & $6.722 \times 10^{-2}$ & $1.365 \times 10^{-1}$ \\
\hline
\end{tabular}

(a)

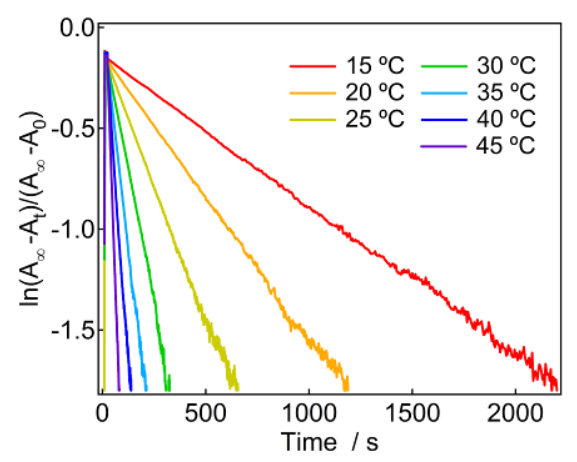

(b)

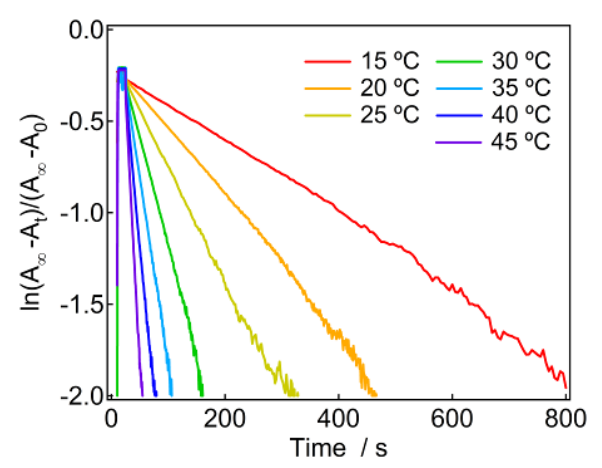

Figure S46. First order kinetic profiles of (a) $\mathbf{1 - Y}$ and (b) $\mathbf{2 - Y}$ monitored at $490 \mathrm{~nm}$ in degassed benzene $\left(2.0 \times 10^{-5} \mathrm{M}\right)$.

The measurements were performed in the temperature range from $15^{\circ} \mathrm{C}$ to $45^{\circ} \mathrm{C}$. 
(a)

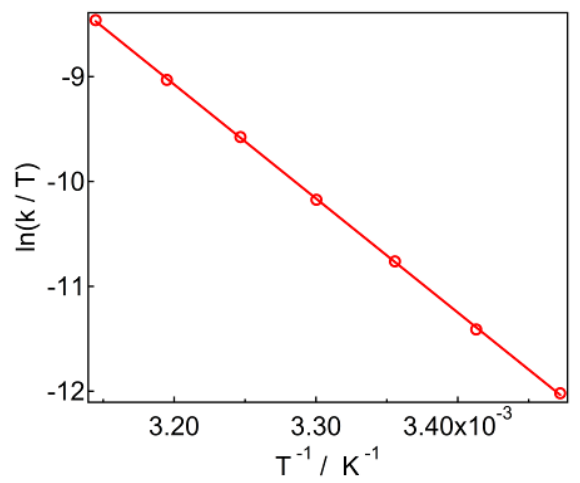

(b)

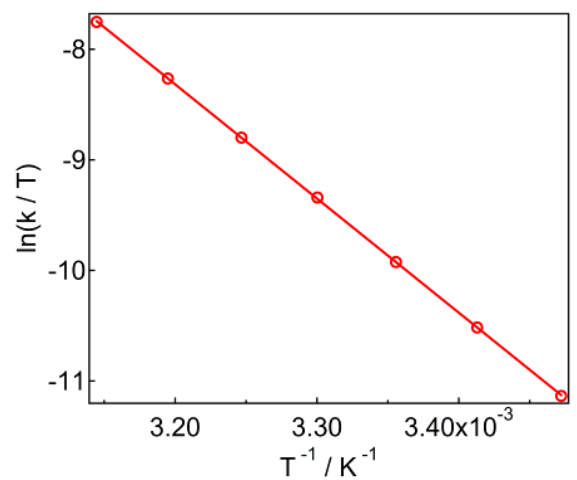

Figure S47. Eyring plot for the thermal back-reaction of (a) 1-Y and (b) 2-Y.

Table S5. Half-Lives and the activation kinetic parameters of $\mathbf{1}$ and $\mathbf{2}$ in benzene at $298 \mathrm{~K}$

\begin{tabular}{ccccc}
\hline compound & $\tau_{1 / 2} / \mathrm{s}$ & $\Delta \mathrm{H}^{\ddagger} \mathrm{kJ} \mathrm{mol}^{-1}$ & $\Delta \mathrm{S}^{\ddagger} \mathrm{J} \mathrm{K}^{-1} \mathrm{~mol}^{-1}$ & $\Delta \mathrm{G}^{\ddagger} \mathrm{kJ} \mathrm{mol}^{-1}$ \\
\hline $\mathbf{1}$ & 109.8 & 90.4 & 16.4 & 85.5 \\
$\mathbf{2}$ & 47.5 & 85.9 & 8.03 & 83.5 \\
\hline
\end{tabular}




\section{Solvent Dependence of Thermal Back Reaction}

(a)

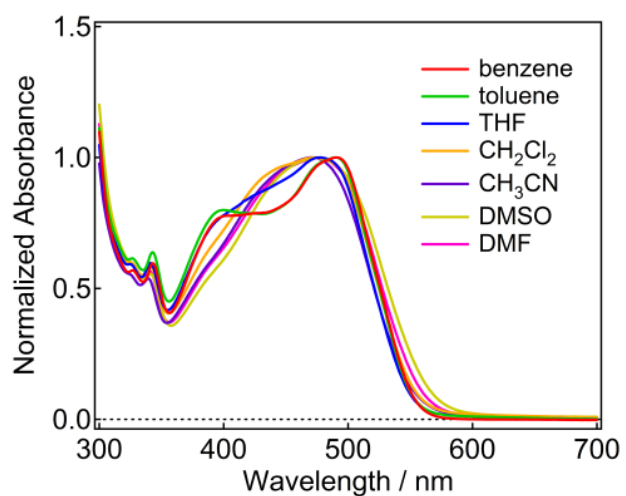

(b)

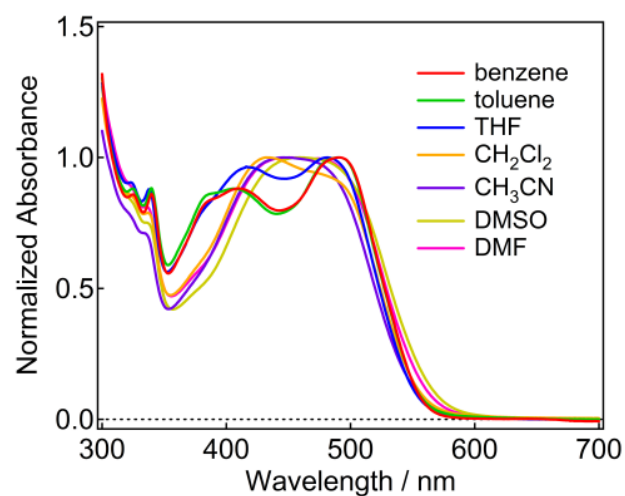

Figure S48. Absorption spectra of (a) 1-O and (b) 2-O in various solvents.

(a)

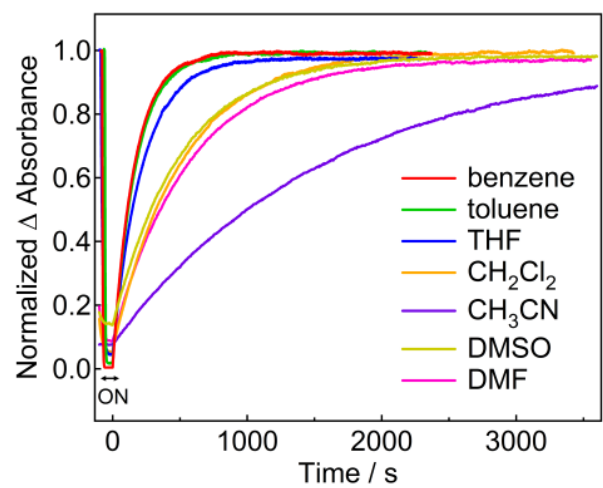

(b)

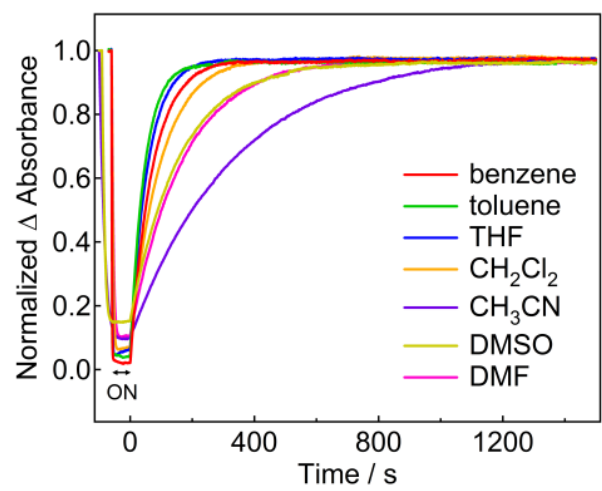

Figure S49. Thermal back reactions monitored at $490 \mathrm{~nm}$ of (a) 1-Y and (b) 2-Y generated from the orange forms upon irradiation with visible light $(525 \mathrm{~nm}, 83 \mathrm{~mW})$ in various solvents at $298 \mathrm{~K}$.

Table S6. Half-Lives of 1-Y and 2-Y in various solvents at $298 \mathrm{~K}$

\begin{tabular}{ccc}
\hline compound & $\tau_{1 / 2} / \mathrm{s}(\mathbf{1})$ & $\tau_{1 / 2} / \mathrm{s}(\mathbf{2})$ \\
\hline benzene & 109.8 & 47.5 \\
toluene & 117.9 & 31.5 \\
$\mathrm{THF}$ & 151.6 & 38.9 \\
$\mathrm{CH}_{2} \mathrm{Cl}_{2}$ & 356.2 & 66.8 \\
$\mathrm{CH}_{3} \mathrm{CN}$ & 1098.1 & 229.2 \\
$\mathrm{DMSO}$ & 349.1 & 109.2 \\
$\mathrm{DMF}$ & 391.5 & 113.6 \\
\hline
\end{tabular}




\section{References}

S1. Nakano, E.; Mutoh, K.; Kobayashi, Y.; Abe, J. Electrochemistry of Photochromic [2.2]Paracyclophane-Bridged Imidazole Dimers: Rational Understanding of the Electronic Structures. J. Phys. Chem. A. 2014, 118 (12), 2288-2297.

S2. Hatano, S.; Horino, T.; Tokita, A.; Oshima, T.; Abe, J. Unusual Negative Photochromism via a Short-Lived Imidazolyl Radical of 1,1'-Binaphthyl-Bridged Imidazole Dimer. J. Am. Chem. Soc. 2013, 135 (8), 3164-3172.

S3. Relm, D.; Weller, A. Kinetics of Fluorescence Quenching by Electron and H-Atom Transfer. Israel J. Chem. 1970, 8, $259-271$. 